\begin{tabular}{|c|c|c|c|}
\hline Eiszeitalter u. Gegenwart & $\mathbf{4 4}$ & $79-93$ & Hannover 1994 \\
\hline
\end{tabular}

\title{
Thermolumineszenz-Datierungen an Lössen des Tönchesberges aus der Osteifel
}

\author{
MANFRED FRECHEN*) \\ Pleistocene, Weichselian, Saalian, Stratigraphy, loess, thermoluminescence dating, Toenchesberg, \\ East Eifel, Germany
}

\begin{abstract}
Kurzfassung: Anhand von 61 Proben wurden die Grenzen der Anwendbarkeit der Thermolumineszenz(TL)-Datierungsmethode für Lößdeckschichten des TönchesbergSchlackenkegels aus der Osteifel untersucht. Zur Bestimmung der akkumulierten Dosis kamen sowohl die Regenerierungs-Methode als auch die Additive Methode und für einige wenige Proben die R-Gamma-Methode zur Anwendung. Die geologisch-stratigraphische Verläßlichkeit von TL-Altern für die letzten 200000 Jahre wurde anhand von 126 voneinander unabhängigen TL-Daten untersucht. Die TL-Datierungen an Lössen des Profils Tönchesberg sind bis zu einem Alter von etwa 90 ka in guter Übereinstimmung mit den geologisch erwarteten Altern. ${ }^{+1} \mathrm{Ar} /{ }^{\prime \prime} \mathrm{Ar}$-Datierungen von Tephren mit einem physikalischen Alter von $238 \pm 20 \mathrm{ka}$ und $243 \pm 65 \mathrm{ka}$ unterstützen die Vorstellung, daßs signifikante TL-Altersunterbestimmungen für die Proben unterhalb des letztinterglazialen Bodens vorliegen. Die lößstratigraphische Abfolge und malakozoologische Untersuchungen implizieren jedoch, daß die "Ar/3" $\mathrm{Ar}$-Datierungen altersüberbestimmt sind. Die mit der Additiven Methode kalkulierten TL-Alter für vorletztglaziale Lösse sind größer als bei Verwendung der Regenerierungs-Methode. Jedoch nehmen die TL-Alter weder mit der einen noch mit der anderen Methode außerhalb der Mutungsintervalle zum Liegenden hin zu. Neben physikalischen Gründen kann eine rasche Lößakkumulation während der jüngeren Saale-Kaltzeit als Grund für die nicht erkennbare Zunahme der TL-Alter angenommen werden.
\end{abstract}

TL-Daten von mehr als 100000 Jahren werden im allgemeinen durch eine wissenschaftlich fragwürdige Auswahl von Meßdaten sowie Anpassung von Meßbedingungen und Meßparametern ermittelt, um geologischen Altersabschätzungen oder unabhängigen Datierungen zu genügen. Die Unabhängigkeit als Grundprinzip einer Datierungsmethode ist mithin für diesen Altersbereich bei der TL nicht gegeben.

\section{[Thermoluminescence dating of upper Pleistocene loess deposits from Toenchesberg, East Eifel, Germany]}

\footnotetext{
Abstract: 61 samples were investigated from the well studied loess section at Tönchesberg, a scoria cone of the East Eifel Volcanic Field. The samples were investigated by the

*) Anschrift des Verfassers: Dr. M. Frechen, Abt. Quartärgeologie, Geologisches Institut der Universität zu Köln, Zülpicher Str. 49, 50674 Köln
}

additive dose method, regeneration method and a few samples by the partial bleach method. 126 independent TL ages were estimated in order to study the geological stratigraphic reliability of thermoluminescence dating over the last 200000 years.

At Tönchesberg the TL ages up to $90 \mathrm{ka}$ and just within the dating uncertainty at $120 \mathrm{ka}$ are in good agreement with the independently inferred stratigraphy and the expected geological ages. "Ar ${ }^{20} \mathrm{Ar}$-dates at $238 \pm 20 \mathrm{ka}$ and at $243 \pm 65 \mathrm{ka}$ from the volcanic tephra underlying the loess and of the scoria cone at $202 \pm 14 \mathrm{ka}$ seem to imply significant TL age underestimation for the lowest 10 samples underneath the last interglacial soil. However, the aspects of soil stratigraphy and faunal remains suggest that the ${ }^{+0} \mathrm{Ar} /{ }^{30} \mathrm{Ar}$ dates may be overestimated.

The TL ages are somewhat larger for the additive dose technique than for the regeneration technique. However, the clates do not increase significantly with depth for either technique beyond around 90-120 ka. Rapid loess accumulation during the upper part of the penultimate glaciation may explain the not recognizable increase of TL ages with depth. TL dalc's exceeding $100 \mathrm{ka}$ using partial bleach and total bleach procedures may give the impression that the age underestimation problem is now avoidable by using different thermal treatments and a blue pass filter. But the fundamental principle of a dating method of independence is not yet achieved for this dating range.

\section{Einführung}

Obwohl seit Ende der 60er Jahre die Thermolumineszenz(TL)-Methode zur Datierung von Keramiken und Sedimenten angewendet wird, sind viele Fragen bezüglich des Datierungsmodells und der Verläßlichkeit der Daten noch offen. Dennoch sind mit TLDatierungen, unter der Voraussetzung von großen Probenzahlen und engem Beprobungsabstand, zuverlässige und relativ genaue Chronologien letztglazialer Löß/Paläoboden-Abfolgen erhalten worden. Im Idealfall ermöglichen diese radiometrischen Altersbestimmungen regionale und überregionale Korrelationen von Löß-/Paläoboden-Abfolgen.

Unter Thermolumineszenz wird ganz allgemein eine Leuchterscheinung von Stoffen verstanden, die beim Erhitzen neben der Planckschen Strahlung entsteht. 
Sie beruht auf der Fähigkeit vieler Minerale, z. B. Quarz, Feldspat und Zirkon, bei der Absorption von energiereicher Strahlung einen Teil der Anregungsenergie in Form von potentieller Energie zu speichern. Energiereiche, ionisierende Strahlung gibt es überall. Sie stammt aus dem radioaktiven Zerfall von Isotopen der ${ }^{238} \mathrm{U}-$ - ${ }^{235} \mathrm{U}$ - und ${ }^{232} \mathrm{Th}$-Zerfallsreihen, dem Zerfall von ${ }^{40} \mathrm{~K}$ und untergeordnet einiger seltener vorkommender Radioelemente (beispielsweise ${ }^{8-} \mathrm{Rb}$ ) sowie der kosmischen Strahlung.

Das Datierungsprinzip beruht auf dem zeitlichen Anwachsen eines "Strahlenschadens", der akkumulierten Dosis, nach der Sedimentation. Durch Zuführung von Energie (Hitze, Licht) können diese "Strahlenschäden" beseitigt werden. Dabei kommt es zur Emission von Photonen, deren Lichtintensität im Labor gemessen wird. Sie ist proportional zur Strahlendosis, die das Mineral im Laufe der Zeit gespeichert hat (vgl. AitKen 1985; FreChen 1991a). Das TL-Alter berechnet sich dann, vereinfacht ausgedrückt, aus dem Quotienten von akkumulierter und jährlicher Dosis.

Mit Hilfe der TL lassen sich drei datierbare Ereignisse unterscheiden:

1. Zeitpunkt der Sedimentation (z. B. Lösse, Dünensande, fluviatile und glaziale Sedimente)

2. Zeitpunkt der Kristallisation (z. B. Tephren, Schlacken, Laven, Neubildungen von Mineralen)

3. Zeitpunkt der Abkühlung (z. B. Keramiken, Feuerstellen, Sedimente aus Frittungszonen).

Bei äolischen Sedimenten wird im Idealfall der Zeitpunkt der letzten Sonnenlicht-Exposition bestimmt. Die "TL-Uhr" des Ursprungsmaterials wird während des äolischen Transportes unter anderem durch das UV-Licht der Sonne bis auf Null oder bis auf einen unbleichbaren Restbetrag zurüickgestellt. Inwieweit TLDaten das wirkliche Sedimentationsalter von Lössen wiedergeben, hängt vor allem davon ab, ob der äolische Staub während des Transportes genügend Zeit hatte, um vollständig gebleicht zu werden und in welchem Umfange die Lösse in der Folgezeit durch Solifluktion oder andere Umlagerungsprozesse beeinflußt worden sind. Untersuchungen an rezenten Lössen aus Alaska zeigen eine bis auf einen nicht bleichbaren Rest vollständige Rückstellung der "TLUhr" (BERGER 1990: 12396). Für die Korngrößenfraktion 4-11 $\mu \mathrm{m}$ von Lössen des Tönchesberges war der Nullstellungprozeß nach einer Belichtung mit natürlichem Sonnenlicht (sonniger Augusttag in Köln) nach 12 bis 15 Stunden abgeschlossen. Sind äolische oder fluviatile Sedimente nicht vollständig durch Sonnenlicht gebleicht, führt dies zu Altersüberschätzungen bzw. zu Mischaltern, die keinen oder nur sehr geringen Aussagewert besitzen.

Die ersten TL-Alter aus der Bundesrepublik Deutschland bestimmten WINTLE \& BRUNNACKER (1982) für Lösse aus Wallertheim, Rheinhessen. Seitdem wurden für mitteleuroäische Löß-Paläoboden-Abfolgen zahlrei- che TL-Datierungsstudien durchgeführt (u. a. WINTLE 1985; ZÖLler, Stremme \& WAGNer 1988; JuVIGNE \& WINTLE 1988; AKTAS \& FreCHEN 1991; FreChEN 1991a, JANnOtTA 1991; Buschbeck et al. 1992; Frechen, BrüCKNER \& RADTKE 1992).

Für das Profil Tönchesberg liegen interdisziplinäre Forschungsresultate vor, darunter auch Ergebnisse unabhängiger Datierungsmethoden. Es gibt somit ausreichend Vergleichsmöglichkeiten für TL-Alter. Aufgrund der dichten TL-Beprobung und der systematischen Bearbeitungsweise wurden der chronologische, paläoklimatische und paläoökologische Informationsgehalt des Profils Tönchesberg erheblich erweitert (BOenigk \& Frechen 1991 und im Druck).

\section{Abriß des Forschungsstandes/ Datierungs- problematik}

Ein detaillierter Abriß des Forschungsstandes von TLAltersbestimmungen an Lössen ist bereits an anderer Stelle erfolgt (vgl. Wintle 1990, Frechen 1991a), so daß hier nur neuere Arbeiten exemplarisch diskutiert zu werden brauchen.

Die grundlegenden Untersuchungen von WintLE (1985) und Debenham (1985) bezüglich des Problems von TL-Altersunterbestimmungen werden durch die eigene Arbeit für die Profile Ariendorf und Tönchesberg aus dem Mitteltheingebiet und durch FRECHEN, BRÜCKNER \& RADTKE (1992) für das Profil Rheindahlen aus dem Niederrheingebiet bestätigt. Verläßliche TLAlter sind mit der TL-Analyse hoher Probenzahlen bis zu einer Datierungobergrenze von 90 bis 120 ka (1 ka $=1000$ Jahre) zu erhalten. Da TL-Datierungen äußerst arbeitsintensiv sind, wird oft versucht, Löß-Paläoboden-Abfolgen mit wenigen Proben zu datieren. Die geologisch-stratigraphische Verläßlichkeit der auf diese Weise erhaltenen physikalischen Alter ist meist nicht gegeben. Stichprobenartige Untersuchungen können zwar stratigraphisch scheinbar konsistente physikalische Alter bieten, haben aber keine oder nur geringe chronostratigraphische Bedeutung. Erst eine große Anzahl von Daten läßt Schlüsse über den Grad ihrer Genauigkeit zu. Eine chronostratigraphische Parallelisierung von interstadialen Bodenhorizonten innerhalb des letzten Glazials ist mit Daten von Stichproben meist nur eingeschränkt möglich. Dies gilt besonders für Naßböden, Fließerden und kalkhaltige Braunerden, die zahlreich in fast allen Profilbereichen vorkommen.

Die Beurteilung der Zuverlässigkeit von TL-Daten stützt sich in der Hauptsache auf die geologischen und lößstratigraphischen Zusammenhänge. Dies gestattet freilich nur relative Aussagen, die aber ausreichen, um einen Alterswert als möglich, wahrscheinlich oder unwahrscheinlich einzustufen

Zusätzliche Datierungsergebnisse unabhängiger Methoden sind von großem Nutzen. Für die TL-Alter der 
Lößdeckschichten am Tönchesberg bestehen derartige unabhängige Vergleichsmöglichkeiten. Das Alter des Laacher See-Bimses wurde indirekt warvenchronologisch und mit ${ }^{14} \mathrm{C}$-Datierungen bestimmt. Des weiteren liegen ${ }^{40} \mathrm{Ar} /{ }^{39} \mathrm{Ar}$ - und ${ }^{40} \mathrm{~K} /{ }^{40} \mathrm{Ar}$-Daten zwischengeschalteter Tephren und der TönchesbergSchlacken vor, sowie U/Th-Alter von Knochen und Zähnen (vgl. FreChen 1991b: 107).

Derzeit noch ungelöst ist die Frage, ob vorletztglazialer Löß von älteren Lössen mittels TL-Daten unterschieden werden kann. Untersuchungen an Lössen aus Rheindahlen (FRECHEN, BRÜCKNER \& RADTKE 1992: 111, Tabelle 1) und Ariendorf (FRECHEN 1990a: 115) haben gezeigt, daß numerisch höhere Alter als $120 \mathrm{ka}$ bestimmbar sind. Jedoch fehlen bisher grundlegende Untersuchungen, um diese Ergebnisse relativ oder absolut interpretieren zu können. Einen vielversprechenden Versuch lieferte Balescu (1986: 128 ff), die eine relative TL-Chronologie für nordwesteuropäische Lößprofile, darunter Ariendorf (Mittelrhein), aufstellte.

JANNOTTA (1991) untersuchte letztglaziale Löß-/ Paläoboden-Abfolgen aus Holzkirchhausen (NeckarMain-Gebiet) und Bergerhausen (Rheingau). PACKMAN \& GRÜN (1992) datierten Lösse aus Achenheim (Elsaß). Für letztglaziale Lößablagerungen konnten mit Einschränkungen verläßliche und reproduzierbare TL-Alter bestimmt werden. Beide Arbeitsgruppen sehen jedoch derzeit keine Möglichkeit, zuverlässige TL-Alter für vorletztglaziale oder ältere Lösse $\mathrm{zu}$ bestimmen. In Rheinhessen untersuchten BuscHBECK et al. (1992) oberweichselzeitliche Lößablagerungen von Wallertheim und Gräselberg. Die Alter für die Wallertheimer Tephra unterscheiden sich nicht bzw. nur sehr gering von denen der Eltviller Tephra (vgl. Wintle \& BrunNaCKER 1982: 181). Als Grund nehmen BuschBeck et al. (1992: 23) an, daß die obere Tephra in Wallertheim entweder nicht der Eltviller Tuff ist oder daß das Lößmaterial vor der Sedimentation nicht vollständig gebleicht worden ist. Für das Profil Gräselberg stimmen die TL-Alter nicht mit der lößstratigraphischen Interpretation überein. Für den Eltviller Tuff wurden TL-Alter zwischen $21,4 \pm 2,5$ und $24,0 \pm 3 \mathrm{ka}$ und für den Naßboden E2 TL-Alter zwischen $29,3 \pm 3,3$ und $33,0 \pm 3,6$ ka bestimmt. Die ${ }^{14} \mathrm{C}$-Alter von Mollusken aus dem E2-Naßboden von 18,5 $\pm 0,95$ und 21,1 $\pm 1,4 \mathrm{ka}$ (BuschBECK et al. 1992: 20) sind zu unzuverlässig, um zum Vergleich herangezogen zu werden.

Durch die Variation einzelner oder mehrerer Meßparameter ist es möglich, TL-Alter den geologischen Erwartungen anzupassen (vgl. FreCHEN 1991a: 111 ff, 1991b: 101; GEYH 1991: 261f; WinTLE et al. 1993: 568). Eine subjektive, bewußte oder unbewußte Manipulation von TL-Daten ist allein durch die numerische Altersangabe meist nicht überprüfbar. Erst aus dem kompletten Datensatz geht hervor, inwieweit ein Be- arbeiter durch das Weglassen oder Austauschen von Meßwerten eine Genauigkeit bzw. Datierungsobergrenze suggeriert, die eigentlich wissenschaftlich nicht haltbar ist. Es gibt verschiedene mathematische Auswertemodelle, mit denen die akkumulierte Dosis bestimmt werden kann. Statistische Untersuchungen zeigen, daß Fehler bei der Bestimmung der akkumulierten Dosis, sowohl für die Additive Methode als auch für die Regenierungs-Methode, mittels Integralauswertung im Temperaturbereich zwischen 300 und $400^{\circ} \mathrm{C}$ minimierbar sind. Bei einer akkumulierten Dosis (ED=equivalent dose) von 600 Gray schwankt der Betrag des Integrals um $\pm 20 \%$ innerhalb von Wiederholungsmessungen. Für nicht angepaßte Kurven variiert der Betrag der über Plateaus betimmten ED aber um $\pm 110 \%$. Aus den vorliegenden Untersuchungen mit hoher Probenzahl geht hervor, daßs stichprobenartige TL-Datierungen bei Verwendung der Plateauauswertung für die chronostratigraphische Interpretation nicht ausreichen und wenig verläßlich sind (FRECHEN 1991a: 107, 1992: 99). Ebenso unsicher ist die Bestimmung der akkumulierten Dosis über die Peak-Temperatur bei $320^{\circ} \mathrm{C}$ oder $340^{\circ} \mathrm{C}$. Durch Weglassen oder Hinzufügen des höchsten Dosiswertes sind dabei erhebliche Schwankungen, im Extremfall bis zu 40\%, möglich (FreChen 1991a: 33). Dieser Schwankungsbereich impliziert Altersverschiebungen um den gleichen Betrag. Aus diesem Grund sind neun unterschiedliche Dosispunkte mit jeweils $5 \mathrm{Ali}$ quots bei guter Reproduzierbarkeit als Mindestforderung anzusehen.

Für die Löß-/Paläoboden-Abfolge des Tönchesberges liegen TL-Daten von Zöller, CONARD \& HAHN (1991: 408) vor. Für die Proben oberhalb des Eem-Bodens wurden zu ihrer Ermittlung unterschiedliche Temperaturpeaks bei $320^{\circ} \mathrm{C}$ oder $340^{\circ} \mathrm{C}$ zur Bestimmung der akkumulierten Dosis verwendet und ein konstantes Uran-/Thorium-Verhältnis von 1 zu 3,4 angenommen. Für die Proben unterhalb des Eem-Bodens wurden ohne hinreichende Begründung keine konstanten Uran-/Thorium-Verhältnisse für die Dosisratenberechnung angenommen. Eine Einschätzung dieses Datensatzes ist aufgrund dieses nicht begründeten Austausches von physikalischen Meßparametern schwerlich möglich. Es fehlen Erläuterungen, warum die $320^{\circ} \mathrm{C}$ - und $340^{\circ} \mathrm{C}$-Peaks zur Auswertung herangezogen, und warum gerade für die wegen der Datierungsobergrenze problematischen Proben unterhalb des Eem-Bodens eine andere Dosisratenberechnung angewendet worden ist. Wie problematisch eine Variation von Meßparametern sein kann, beschreiben FRECHEN (1991a: 111f) für das Profil Ariendorf und FRECHEN, BRÜCKNER \& RADKTE (1992: 112) am Beispiel des Lößprofils Rheindahlen. Obwohl die TLAlter von ZÖlLER, CONARD \& HAHN (1991: 409) numerisch innerhalb der $1 \sigma$-Abweichung mit denen der vorliegenden Arbeit übereinstimmen, werden sie aus 
den oben beschriebenen Gründen nicht weiter verwendet.

Es überrascht immer wieder, daß auch in neueren Arbeiten der Eindruck vermittelt wird, daß Lösse bis zu mehreren $100 \mathrm{ka}$ mit TL problemlos datierbar sind. Es ist wichtig, dies als noch immer nicht erreichtes Arbeitsziel zu sehen. Berger, Pillans \& Palmer (1992: 403) behaupten, daß Lösse aus Alaska und Neuseeland mit der Regenerierungs-Methode ("total bleach method") bis zu einem absoluten Alter von 800 ka verläßlich datiert worden sind. Die Autoren sind der Meinung, daß die Probleme der Altersunterbestimmungen von polymineralischem Feinkornmaterial (DeBENHAM 1985: 717 ff; WinTle 1985: 730; WinTLE \& PACKMAN 1988: 319; FRECHEN 1991a: 85) durch unterschiedliche Vorheizbedingungen und Verwendung von Blaufiltern (Schott UG 11 und BG28) während des Meßvorganges zu beheben seien. WinTLE et al. (1993) stellen dies in Frage, da durch die Verwendung unterschiedlicher ultravioletter oder blauer Bandpaßfilter kaum differierende akkumulierte Dosen gefunden worden sind (vgl. PACKMAN \& GRÜn 1992: 105).

Das höchste TL-Alter von 730 ka hat eine Standardabweichung von $\pm 250 \mathrm{ka}$. Dies bedeutet, daß das physikalische Alter mit einer Wahrscheinlichkeit von 95\% zwischen 230 und 1230 ka liegt. Die Breite dieses Schwankungsbereichs läßt die Aussage einer TL-Datierungsobergrenze von 800 ka nicht zu.

Berger, Pillans \& Palmer (1992: 404) vertrauen auf den Plateautest als Kriterium für die Zuverlässigkeit bei der Bestimmung der akkumulierten Dosis. Der für die Altersbestimmung von Keramik entwickelte und dort auch aussagekräftige Plateautest erwies sich jedoch selbst bei Untersuchungen mit hoher Probenzahl besonders für ältere Lösse als wenig geeignet und aussagekräftig (Frechen 1991a: 104). Berger, Pillans \& PALMER (1992: 404) unterstreichen, daß für Lößproben aus Neuseeland und Alaska vergleichbare Daten unabhängiger Methoden vorliegen. Ob unabhängig bestimmte, "absolute" Alter verläßlicher sind als andere, bleibt jedoch offen (FreCHEN 1991b: 107). Schließlich ist zu bemerken, daß die älteste Probe (730 $\pm 250 \mathrm{ka}) 5 \mathrm{~m}$ unterhalb der Brunhes/MatuyamaGrenze genommen wurde und für sie trotzdem ein Alter von $800 \mathrm{ka}$ angenommen wird. Berger, PiLlans \& Palmer (1992) nehmen auch keine Stellung zu methodischen Schwierigkeiten, die bei TL-Datierungen von Lössen weltweit in vielen anderen Lößregionen beobachtet worden sind.

Es ist nicht auszuschließen, daß Meßbedingungen und -parameter so angepaßt werden, daß die TL-Alter mit den geologischen Altersabschätzungen oder unabhängigen Daten in Einklang stehen. Es bleibt fraglich, ob nach dem Variieren und Anpassen physikalischer Faktoren TL-Alter noch als physikalisch zuverlässig dargestellt und kritiklos auf einen Altersbereich von bis zu $800 \mathrm{ka}$ übertragen werden dürfen.
Modelle zur Lösung der Problematik von TL-Altersunterbestimmungen sind noch immer unbefriedigend (vgl. FRECHFN 1991a: 105; JANNOTTA 1991: 67; XIE \& AITKEN 1991: 21; WINTLE 1990: 394 ff).

Neben laborspezifischen Fehlermöglichkeiten existieren die methodischen Schwierigkeiten des Kurzund Langzeit-Fadings, der Sensitivitätsveränderungen und unvollständigen Bleichung, aber auch dosimetrische Probleme und offene Systeme treten auf. Diese Probleme sind teilweise mit mehr oder weniger großem experimentellen Aufwand lösbar oder zumindest in ihrer Größenordnung abschätzbar (ArTKEN 1985: 135ff, FRECHEN 1991a: 92 ff).

Der Fehlerbereich bei der Bestimmung des Uran-, Thorium- und Kaliumgehaltes mit der Gammaspektrometrie liegt bei etwa 5 - 8\%. Andere analytische Methoden wie Neutronenaktivierung, Alpha-Counting, Thick Source Beta Counting oder Flammenphotometrie sind nicht genauer. Am genauesten ist die Bestimmung der Dosisrate durch direkte Messung der Alpha-, Beta- und Gammastrahlung und Vergleich mit Ergebnissen der Neutronenaktivierungsanalyse.

Die Dosisraten für die Proben des Tönchesberges liegen zwischen 3,6 und 4,9 Gray pro 1000 Jahre, also im Wertebereich von 4-6 Gray pro 1000 Jahre, die WinTLE (1990: 386) als typisch für Lösse aller Kontinente ansieht. Die jährliche Dosis kann indessen immer noch genauer bestimmt werden als die akkumulierte Dosis.

Die Elemente Uran, Thorium und Radon können nämlich im Laufe des Alterns der Sedimente durch Grundwasserzirkulation in das oder aus dem Sediment migrieren. Die Mobilität dieser Elemente ist in feinkörnigen, schluffigen Sedimenten geringer als in grobkörnigen, sandigen Sedimenten. Finden solche Prozesse statt, entstehen radioaktive Ungleichgewichte, die für die Proben des Tönchesberges nicht festgestellt wurden.

Da in Wasser radioaktive Strahlung teilweise absorbiert wird, ist der Wassergehalt des Sediments bei der Berechnung der Dosisrate zu berücksichtigen. Veränderungen der Feuchte in der geologischen Vergangenheit stellen aber einen nur schwer kalkulierbaren Fehler dar.

\section{Methodik}

Die einzelnen Arbeitsschritte von der Probennahme bis zur geologischen Interpretation der in dieser Arbeit vorgestellten Ergebnisse sind bei FRECHEN 1991a: 24ff) ausführlich beschrieben.

Für die Lößdeckschichten des Tönchesberges wurden erstmalig TL-Analysen mit hoher Probenzahl durchgeführt (FRECHEN 1990b: 56f, 1991a: 78 ff). 61 Proben wurden mit der Regenerierungs-Methode, der Additiven Methode und der R-Gamma-Methode analysiert. Die Auswertung lieferte 126 TL-Alter. Außer- 
dem haben sechs verschiedene TL-Labore dieselben Proben aus dem obersten Bereich des unterweichselzeitlichen Schwemmlösses zum Vergleich analysiert (FRECHEN 1991a: 91).

Die akkumulierte Dosis wurde für die polymineralische Feinkornfraktion 4-11 $\mu \mathrm{m}$ bestimmt (Abb. 1). Die Anwendung von drei Methoden, wegen des hohen Arbeitsaufwandes ist sicherlich ungewöhnlich, erschien aber aufgrund der Schwierigkeiten bei der Bestimmung der akkumulierten Dosis erforderlich.
Der Schnittpunkt der additiven Aufbaukurve mit der TL-Intensität des unbleichbaren Rests $\left(\mathrm{I}_{0}\right)$ ergab die akkumulierte Dosis (Abb. 1). Bei der R-Gamma-Methode wurden zwei Aliquotserien additiv bestrahlt. Anschließend wurde eine Aliquotserie mit einer UVLampe für zwei Minuten belichtet. Der Schnittpunkt der additiven mit der teilweise belichteten Aufbaukurve ergab die akkumulierte Dosis (Abb. 1). Mittels der R-Gamma-Methode sollen sich auch unvollständig gebleichte Sedimente analysieren lassen. Für die

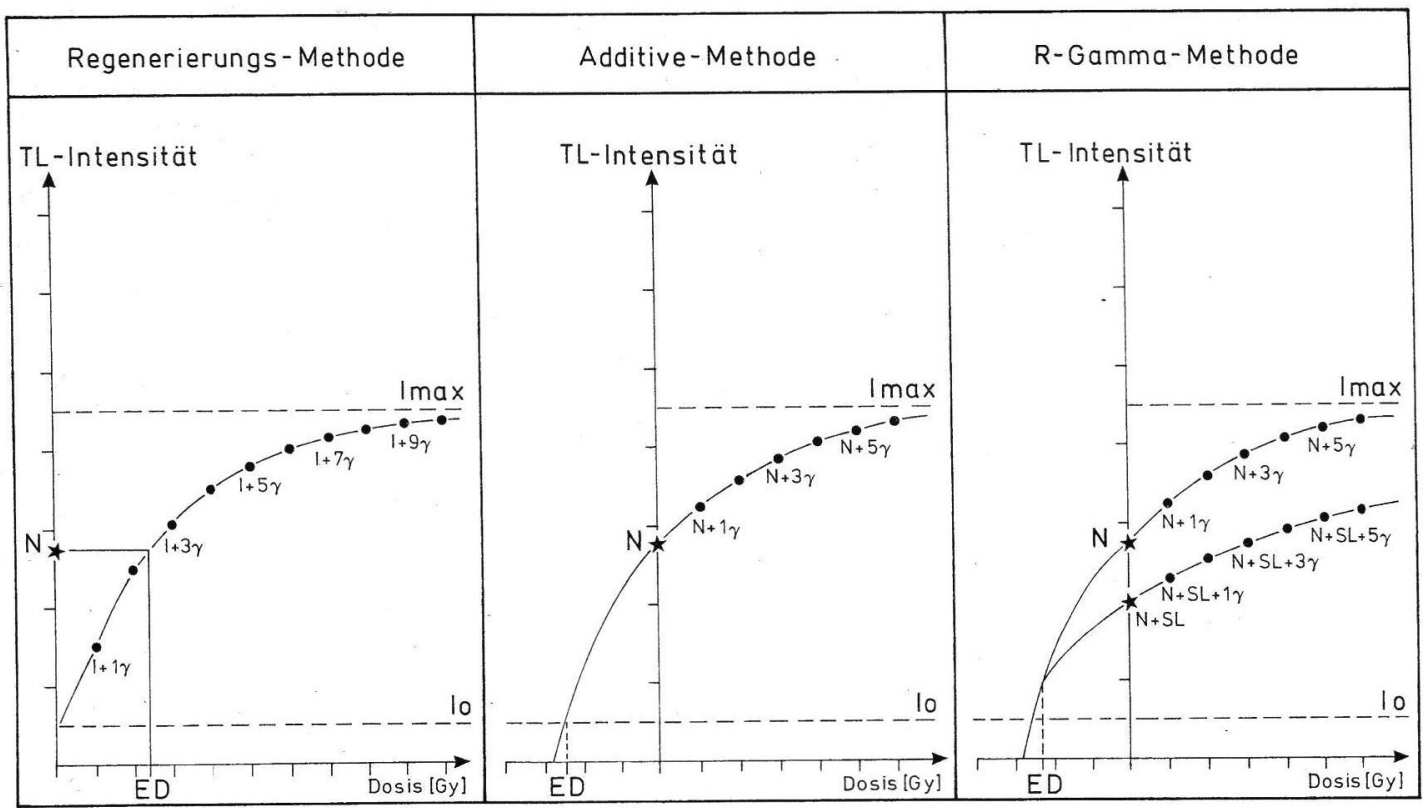

Abb. 1: Methoden zur Bestimmung der akkumulierten Dosis (ED): Regenerierungs-Methode (REGEN), Additive Methode (ADD) und R-Gamma-Methode (R-Gamma). Erläuterungen im Text.

Fig. 1: Methods suitable for determination of equivalent dose (ED): regeneration method (REGEN), additive dose method (ADD) and partial bleach method (R-Gamma). For explanation see text.

Die Regenerierungs-Methode (REGEN) und die Additive Methode (ADD) wurden beide unter der Annahme angewendet, daß das Sonnenlicht die Lumineszenz der Minerale während des äolischen Transportes bis auf einen nicht bleichbaren Rest reduziert hat. Bei beiden Methoden wurde versucht, die Vorgänge in der Natur im Labor nachzuahmen. Die Nullstellung durch Sonnenlicht wurde beispielsweise durch das UV-Licht einer Osram Ultravitalux 300 Watt-Lampe oder natürliches Sonnenlicht und das Anwachsen der akkumulierten Dosis durch künstliche Bestrahlung nachgeahmt (Abb. 1). Bei der Regenerierungs-Methode wurden die Aliquots zunächst bis auf den unbleichbaren Rest belichtet und anschließend mit verschiedenen Dosen bestrahlt. Der Schnittpunkt der Aufbaukurve mit der natürlichen TLIntensität der Probe (N) ergab die akkumulierte Dosis. Bei der Additiven Methode wurden Aliquots sukzessive mit unterschiedlichen Gammadosen bestrahlt. letztgenannte Methode fehlen allerdings noch systematische Vergleiche und Untersuchungen, so daß die vier auf diese Weise erhaltenen TL-Alter in dieser Arbeit nicht diskutiert werden.

Für alle drei Methoden wurde, der geringeren Streuung der Einzelwerte wegen, eine exponentielle Regression bevorzugt und das Integral des Temperaturbereichs zwischen 300 und $400^{\circ} \mathrm{C}$ ausgewertet. Es wurden jeweils mindestens 9 verschiedene Dosispunkte für die Kalkulation der Daten verwendet. Die Plateau-Methode wurde wegen erheblicher Variationen der Ergebnisse nicht angewendet. Selbst der angewendete Plateau-Test lieferte keine verläßliche, physikalische Basis für die TL-Datierung.

Die jährliche Dosis wurde mit einem NaI-Gammaspektrometer im Gelände und mit einem Ge-Gammaspektrometer im Labor gemessen. Dosimetrische Vergleichsuntersuchungen erfolgten mit der Neutronenaktivierung (KFA Jülich) und der Flammenpho- 
tometrie (Godwin Laboratory, Cambridge) sowie Alpha-Counting (Institute of Earth Studies, Aberystwyth). Für die Berechnung des TL-Alters wurden ausschließlich die Analysenergebnisse der gammaspektrometrischen Labor- und Geländeuntersuchungen verwendet.

Für die Proben des Tönchesberges stimmen die sich entsprechenden TL-Daten der Regenerierungs-Methode und der Additiven Methode innerhalb von $\pm 15 \%$ überein (Tabelle 1).

Erwähnenswert sind die zwar geringen, aber systematischen Unterschiede in bezug auf die akkumulierte Dosis und somit auf das Alter. Die TL-Alter der
Additiven Methode des oberweichselzeitlichen Lösses (ED zwischen 50 und 100 Gray) sind um 15\% kleiner als die Regenerierungs-Methode. Dieser Unterschied könnte aufgrund von Sensitivitätsveränderungen durch das künstliche Belichten der Lösse mit der UV-Lampe entstanden sein. Dafür sprechen die Ergebnisse der von Frechen (1991a: 92ff) durchgeführten Experimente zu Sensitivitätsveränderungen. Nimmt man zum Vergleich das Alter des Laacher See Bimses heran, so erscheinen die TL-Alter der Additiven Methode geologisch wahrscheinlicher als die Alter der Regenerierungs-Methode. Bis zu den unterweichselzeitlichen Lössen sind die TL-Alter beider

Tabelle 1: Die Dosisrate wurde über gammaspektrometrische Sedimentanalysen bestimmt. Für die Berechnung der akkumulierten Dosis wurde das Integral des Temperaturbereichs zwischen $300-400^{\circ} \mathrm{C}$ sowohl für die Regenerierungs-Methode (REGEN) als auch für die Additive Methode (ADD) zugrunde gelegt.

\begin{tabular}{|c|c|c|c|c|c|}
\hline & & REGEN & REGEN & ADD & ADD \\
\hline Probe & Dosisrate & Akk. Dosis & TL-Alter & Akk. Dosis & TL-Aiter \\
\hline & [Gy/ka] & [Gy] & [ka] & [Gy] & [ka] \\
\hline$T 1$ & 4,1746 & $61,9 \pm 2,0$ & $14,8 \pm 1,8$ & $53,6 \pm 3,2$ & $13,2 \pm 1,8$ \\
\hline 12 & 4,5598 & $66,4 \pm 0,8$ & $14,3 \pm 1,5$ & $54,7 \pm 1,4$ & $12,0 \pm 1,3$ \\
\hline T3 & 4,0662 & $65,6 \pm 3,6$ & $15,7 \pm 1,8$ & $51,8 \pm 9,9$ & $12,7 \pm 2,8$ \\
\hline T4 & 4,1264 & & & & \\
\hline T5 & 4,4026 & $63,6 \pm 2,6$ & $14,1 \pm 1,5$ & $54,7 \pm 2,4$ & $12,4 \pm 1,4$ \\
\hline TE & 4,4688 & $66,6 \pm 1,4$ & $14,6 \pm 1,5$ & $59,1 \pm 2,0$ & $13,2 \pm 1,4$ \\
\hline T7 & 4,3162 & $66,2 \pm 2,6$ & $15,0 \pm 1,6$ & $59,1 \pm 1,0$ & $13,7 \pm 1,4$ \\
\hline T8 & 4,1027 & $66,6 \pm 4,3$ & $15,8 \pm 1,9$ & $58,3 \pm 3,0$ & $14,2 \pm 1,6$ \\
\hline T9 & 4,2451 & $71,2 \pm 2,0$ & $16,4 \pm 1,7$ & $57,9 \pm 2,4$ & $13,6 \pm 1,5$ \\
\hline T10 & 4,269 & $76,9 \pm 3,0$ & $17,5 \pm 1,9$ & $65,0 \pm 4,7$ & $15,1 \pm 1,9$ \\
\hline T11 & 4,3671 & $93,1 \pm 7,5$ & $20,8 \pm 2,8$ & $108,1 \pm 10,1$ & $24,7 \pm 3,5$ \\
\hline$T 12$ & 3,7667 & $148,2 \pm 5,5$ & $38,3 \pm 4,3$ & $140,7 \pm 7,1$ & $37,4 \pm 4,4$ \\
\hline T13 & 4,3963 & $163,9 \pm 4,1$ & $35,5 \pm 4,0$ & $164,4 \pm 4,1$ & $37,3 \pm 4,1$ \\
\hline 114 & 3,964 & $171,0 \pm 14,0$ & $42,1 \pm 5,6$ & $127,5 \pm 10,2$ & $32,3 \pm 4,3$ \\
\hline T15 & 3,7248 & $165,8 \pm 2,8$ & $43,3 \pm 4,7$ & $146,3 \pm 3,9$ & $39,2 \pm 4,3$ \\
\hline T16 & 3,9126 & $185,4 \pm 8,5$ & $46,2 \pm 5,3$ & $212,5 \pm 17,0$ & $54,4 \pm 7,2$ \\
\hline$T 17$ & 4,0286 & $226,3 \pm 6,5$ & $54,8 \pm 6,0$ & $208,7 \pm 3,6$ & $51,9 \pm 5,6$ \\
\hline T18 & 4,0348 & $218,8 \pm 4,1$ & $52,9 \pm 5,7$ & $225,1 \pm 3,9$ & $55,8 \pm 6,0$ \\
\hline T19. & 3,9131 & $227,7 \pm 4,5$ & $56,7 \pm 6,0$ & $230,3 \pm 4,1$ & $58,8 \pm 6,3$ \\
\hline T20 & 4,235 & $234,0 \pm 3,9$ & $54,0 \pm 5,6$ & $224,5 \pm 2,2$ & $53,1 \pm 5,5$ \\
\hline T21 & 3,8517 & $253,8 \pm 9,7$ & $64,2 \pm 7,2$ & $270,4 \pm 8,5$ & $70,1 \pm 7,7$ \\
\hline T22 & 4,255 & $235,4 \pm 6,3$ & $54,1 \pm 5,7$ & $246,1 \pm 7,7$ & $57,8 \pm 6,2$ \\
\hline$T 23$ & 3,9649 & $236,8 \pm 7,3$ & $58,3 \pm 6,4$ & $238,0 \pm 42,1$ & $60,0 \pm 12,3$ \\
\hline T24 & 3,8018 & $246,5 \pm 8,3$ & $63,2 \pm 7,0$ & $212,3 \pm 7,3$ & $55,8 \pm 6,2$ \\
\hline T25 & 3,8491 & $253,8 \pm 8,3$ & $64,3 \pm 7,1$ & $264,9 \pm 9,5$ & $68,9 \pm 7,7$ \\
\hline 126 & 3,7545 & $252,6 \pm 20,2$ & $65,5 \pm 8,8$ & $283,6 \pm 22,7$ & $75,6 \pm 10,2$ \\
\hline T27 & 4,2725 & $322,2 \pm 6,1$ & $73,7 \pm 7,7$ & $322,6 \pm 5,9$ & $75,6 \pm 7,9$ \\
\hline T28 & 4,2753 & $332,5 \pm 4,9$ & $76,0 \pm 7,9$ & $356,4 \pm 7,1$ & $83,3 \pm 8,7$ \\
\hline T50 & 3,6897 & $287,8 \pm 11,7$ & $76,0 \pm 8,7$ & $260,1 \pm 17,8$ & $70,5 \pm 8,1$ \\
\hline T29 & 4,4976 & $387,4 \pm 14,0$ & $84,3 \pm 9,3$ & $428,1 \pm 13,4$ & $95,2 \pm 10,4$ \\
\hline T30 & 3,9814 & $373,2 \pm 7,5$ & $91,4 \pm 9,8$ & $368,6 \pm 10,3$ & $92,7 \pm 10,1$ \\
\hline T31 & 3,7974 & $382,1 \pm 5,1$ & $98,1 \pm 10,3$ & $424,6 \pm 21,7$ & $111,9 \pm 13,0$ \\
\hline T100 & 3,9946 & $394,0 \pm 16,8$ & $92,2 \pm 9,7$ & $394,0 \pm 30,4$ & $96,6 \pm 11,8$ \\
\hline T32 & 4,0785 & $367,0 \pm 20,6$ & $87,8 \pm 10,4$ & $395,3 \pm 15,2$ & $96,9 \pm 10,7$ \\
\hline T33 & 4,2784 & $380,7 \pm 35,4$ & $87,0 \pm 12,1$ & $415,9 \pm 58,5$ & $97,2 \pm 16,9$ \\
\hline T34 & 4,0841 & $423,2 \pm 20,6$ & $101,2 \pm 11,6$ & $452,4 \pm 21,7$ & $110,7 \pm 12,6$ \\
\hline T35 & 4,3007 & $424,4 \pm 34,0$ & $96,5 \pm 12,5$ & $558,6 \pm 44,7$ & $130,0 \pm 16,9$ \\
\hline T36 & 4,1751 & $413,3 \pm 17,8$ & $96,7 \pm 10,8$ & $411,9 \pm 48,4$ & $98,7 \pm 15,4$ \\
\hline T37 & 4,0909 & $386,0 \pm 4,5$ & $92,1 \pm 9,6$ & $449,1 \pm 11,5$ & $109,8 \pm 11,7$ \\
\hline 138 & 3,8293 & $378,7 \pm 10,7$ & $96,4 \pm 10,5$ & $367,2 \pm 9,7$ & $95,8 \pm 10,4$ \\
\hline T39 & 4,0207 & $379,9 \pm 12,4$ & $92,2 \pm 10,1$ & $415,7 \pm 39,3$ & $103,5 \pm 14,6$ \\
\hline T40 & 4,1626 & $394,3 \pm 11,1$ & $92,5 \pm 10,0$ & $393,3 \pm 7,3$ & $94,4 \pm 10,0$ \\
\hline T41 & 4,0133 & $346,1 \pm 14,2$ & $84,1 \pm 9,5$ & $362,7 \pm 32,4$ & $90,5 \pm 12,5$ \\
\hline T42 & 3,7892 & $361,7 \pm 8,9$ & $93,0 \pm 10,1$ & $367,0 \pm 30,4$ & $96,9 \pm 13,0$ \\
\hline T43 & 4,8755 & $345,7 \pm 9,3$ & $69,5 \pm 7,4$ & $370,4 \pm 24,1$ & $75,9 \pm 9,3$ \\
\hline T44 & 3,5949 & $339,4 \pm 10,3$ & $91,9 \pm 10,3$ & $362,1 \pm 9,5$ & $100,7 \pm 11,2$ \\
\hline T45 & 4,3245 & $328,9 \pm 26,3$ & $74,3 \pm 9,8$ & $400,1 \pm 32,0$ & $92,5 \pm 12,3$ \\
\hline T60 & 4,5014 & $452,4 \pm 16,2$ & $100,5 \pm 9,2$ & $444,3 \pm 18,9$ & $98,7 \pm 10,3$ \\
\hline T61 & 4,627 & $405,2 \pm 21,9$ & $85,7 \pm 10,1$ & $411,9 \pm 35,8$ & $89,0 \pm 12,1$ \\
\hline T62 & 4,8308 & $438,1 \pm 22,5$ & $90,7 \pm 9,6$ & $448,8 \pm 19,5$ & $92,9 \pm 11,5$ \\
\hline T63 & 4,7481 & $458,0 \pm 8,1$ & $96,5 \pm 12,8$ & $501,9 \pm 17,4$ & $105,7 \pm 13,4$ \\
\hline 171 & 3,9016 & $460,0 \pm 14,0$ & $117,9 \pm 8,9$ & $481,1 \pm 20,3$ & $123,3 \pm 10,4$ \\
\hline T72 & 4,2333 & $485,8 \pm 14,8$ & $112,1 \pm 12,3$ & $594,9 \pm 46,2$ & $140,6 \pm 18,4$ \\
\hline 173 & 4,3728 & $464,0 \pm 13,2$ & $103,7 \pm 10,6$ & $537,3 \pm 48,4$ & $122,8 \pm 15,7$ \\
\hline T74 & 4,4709 & $514,7 \pm 4,7$ & $112,6 \pm 11,9$ & $521,4 \pm 14,4$ & $116,5 \pm 12,7$ \\
\hline T75 & 4,1698 & $477,1 \pm 14,6$ & $111,7 \pm 12,3$ & $483,1 \pm 44,9$ & $115,8 \pm 16,3$ \\
\hline T76 & 4,506 & $488,8 \pm 6,7$ & $106,2 \pm 11,3$ & $551,3 \pm 17,6$ & $122,3 \pm 13,5$ \\
\hline T77 & 4,572 & $487,0 \pm 23,9$ & $104,2 \pm 10,4$ & $495,2 \pm 6,7$ & $108,3 \pm 11,1$ \\
\hline$T 78$ & 4,386 & $483,7 \pm 40,3$ & $107,8 \pm 14,5$ & $524,0 \pm 49,6$ & $119,5 \pm 16,9$ \\
\hline T79 & 4,6591 & $512,0 \pm 16,8$ & $107,6 \pm 10,2$ & $531,0 \pm 37,5$ & $114,0 \pm 13,2$ \\
\hline T80 & 4,2571 & $506,4 \pm 25,1$ & $116,2 \pm 13,5$ & $504,2 \pm 9,1$ & $118,4 \pm 12,7$ \\
\hline
\end{tabular}


Methoden innerhalb der Mutungsintervalle vergleichbar. Da unsere Ergebnisse der Additiven Methode denen anderer Untersuchungen widersprechen (Rendell \& Townsend 1988: 253), ist es wichtig, derartige kombinierte Untersuchungen an weiteren Lößprofilen zu wiederholen.

\section{Geologische Situation}

Der Tönchesberg gehört zu einer Gruppe kleinerer Vulkane der Osteifel, die während der vorletzten Kaltzeit ausgebrochen sind. Der Schlackenkegelkomplex des Tönchesberges hat eine Ausdehnung von $0,7 \times 0,6 \mathrm{~km}$ und liegt zwischen den Orten Kruft und Ochtendung in der Osteifel (Abb. 2).

Die bearbeiteten Profile A und B liegen innerhalb ei- ner Muldenstruktur zwischen zwei Schlackenkegeln im Nordwesten und Südwesten. Die Schichtbeschreibung der Profile A und B erfolgte durch Becker, Boenigk \& Hentzsch (1989: 6 ff), die des Profils C durch Hentzsch (1990: 42ff). Eine zusammenfassende Interpretation aller bisher vorliegenden Ergebnisse und eine Beschreibung des paläoklimatischen Verlaufs sowie der Veränderungen der Paläoumweltverhältnisse seit der Eruption des Schlackenkegelkomplexes finden sich bei BOENIGK \& FRECHEN (im Druck).

Das Liegende des Vulkans ist nicht aufgeschlossen, so daß keine Rückschlüsse auf die chronostratigraphische Stellung des Ausbruchs gezogen werden können. Oberhalb des vulkanoklastischen Schutts folgen basanitische Tephren des Korretsberges und des Plaid-

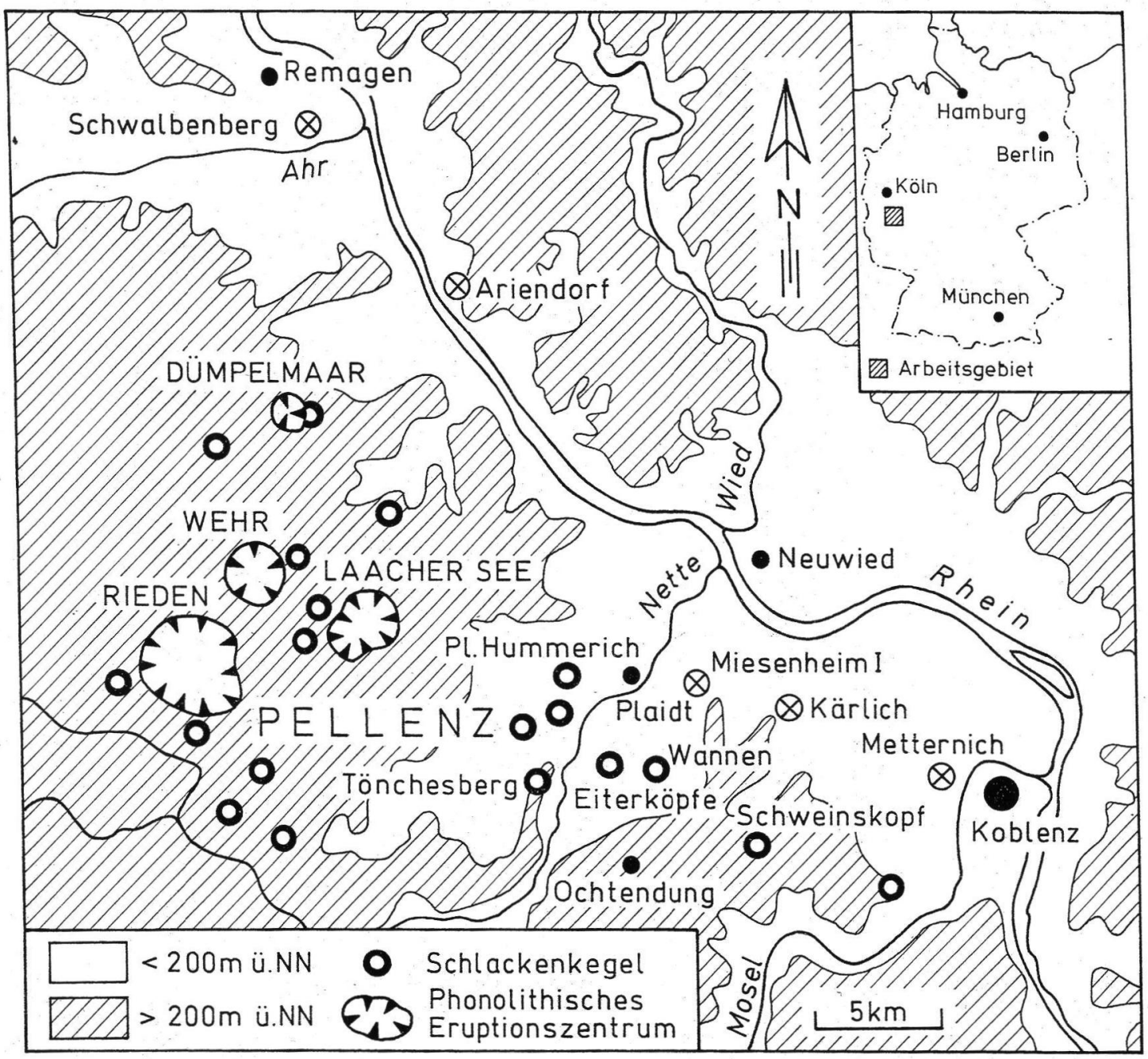

Abb. 2: Lage des Tönchesberges im Vulkanfeld der Osteifel. Kraterprofile sind als schwarze offene Kreise (O) und Umlandprofile als offene Kreise mit Kreuz $(\oplus)$ gekennzeichnet.

Fig. 2: Map showing the location of the loess section Tönchesberg in the East Eifel Volcanic Field, Germany. Crater sections are marked by an open black circle $(O)$, other loess sections by an open circle with a cross $(\oplus)$. 
ter Hummerichs (BOGAARD \& SCHMINCKE 1990: 173). Daraus ergibt sich, daß zunächst der Schlackenkegelkomplex des Tönchesberges ausgebrochen ist, kurze Zeit darauf der des Korretsberges und anschließend der des Plaidter Hummerichs. Darüber folgt ein 0,5 m mächtiges Lößpaket und eine weitere, unter kaltzeitlichen Bedingungen abgelagerte tephritische Aschenschicht, deren Eruptionszentrum bisher noch nicht bekannt ist (BOGAARD \& SCHMINCKE 1990: 179).

Zum Hangenden ist in den Deckschichten ein Solifluktionshorizont mit hohem Anteil an vulkanoklastischem Material aufgeschlossen. Linsen aus hellgrau-bräunlichem, molluskenführenden Schluff sind häufig zwischengeschaltet. Im oberen Bereich hat sich ein Naßboden gebildet. Diskordant darüber folgt eine Löß-/Schwemmlößabfolge, die durch zwei Naßböden und mehrere Vernässungen untergliedert ist. Im Profil C ist der Bt- und Bv-Bereich des interglazialen Bodens wegen des Abbauplanums nicht mehr vorhanden. Jedoch ist etwa $50 \mathrm{~m}$ SSE von Profil C eine Parabraunerde mit humosem Kolluvium und humosen Fließerden aufgeschlossen.

Die Basis der Profile A und B bildet eine gekappte Parabraunerde, die als Rest des Bt-Horizontes des eemzeitlichen Bodens angesprochen wird. Über einer Diskordanz folgen Bodensedimente, die nach BECKER, BOENIGK \& HENZTSCH (1989: 13) aus kolluvial abgeschwemmtem Ah- und Al-Material des letztinterglazialen Bodens bestehen. Die Bodensedimente werden im Sinne von Schönhals, Rohdenburg \& Semmel (1964: 200f) in das Unterweichsel (= Frühweichsel) gestellt. Darüber folgt Schwemmlöß, der von einem Waldsteppenboden (Tschernosem) und von humosen Fließerden überlagert wird. Im Anschluß an diese Umlagerungen ist die oberste humose Fließerde pedogen überprägt worden. Dieser interstadiale Boden wird als zweiter, in situ gebildeter Tschernosem des Unterweichsels angesprochen. Zum Hangenden hin folgt eine weitere humose Fließerde, die von einem geringmächtigen Lößband (Markerlöß i. S. von BECKER, BOENIGK \& HENTZSCH 1989: 10) überlagert wird. Letzteres wird wiederum von einer humosen Fließerde überlagert. Diese wird von BECKER, BOENIGK \& HENTZSCH (1989: 14) als Lehmbröckelsande interpretiert. Darauf folgt ein geringmächtiger Markerlöß, der durch eine humose Fließerde überlagert wird. Über einer Diskordanz liegen Fließerden, die durch zwei schwache interstadiale Bodenbildungen untergliedert werden. Diese Abfolge wird im Sinne von SchÖnhals, Rohdenburg \& SEMmel (1964: 204) dem Mittelweichsel zugerechnet. Die oberste Verbraunung wird von einer Diskordanz gekappt. Ein durch einen schwachen Naßboden untergliedertes Lößpaket wird dem Oberweichsel (= Spätweichsel) zugerechnet. Es wird zum Hangenden hin durch einen interstadialen Boden, der sich während des Alleröds gebildet hat, abgeschlossen. Unmittelbar dar- über liegt der Bims des Laacher See-Ausbruchs. Der holozäne Boden ist abgeschoben.

\section{Diskussion der chronostratigraphischen Ergebnisse}

Die Deckschichten des Tönchesberg-Schlackenkegels bestehen aus Lössen und deren Umwandlungsund Verlagerungsprodukten. Sie werden durch einen mächtigen rotbraunen Bt-Horizont einer Parabraunerde untergliedert. Aufgrund der Deckschichtenabfolge wird die Eruption des Tönchesberg-Schlackenkegels in die vorletzte Kaltzeit gestellt.

Direkt oberhalb der vulkanischen Schlacken liegen unverwitterte Tephraschichten und Löß. Die vorläufigen ${ }^{40} \mathrm{Ar} /{ }^{39} \mathrm{Ar}$-Einzelkristall-Alter der TönchesbergSchlacken von $202 \pm 14$ ka sowie die Alter der Tephren des Korretsberges von $243 \pm 65$ ka (BOGAARD \& SCHMincKe 1990: 178) und des Plaidter Hummerichs von $238 \pm 20 \mathrm{ka}$ (SCHMINCKE \& MERTES 1979: 614) legen das Maximalalter für die Entstehung der Vulkankomplexe fest.

BOGAARD \& SCHMINCKE (1990: 179) ziehen aus den vorliegenden Altern und "stark verwitterten Aschenbänken, die oft Pflanzenabdrücke enthalten", den Schluß, daß der Tönchesberg-Vulkan wie auch der Korretsberg und der Plaidter Hummerich in einer warmzeitlichen Klimaphase ausbrachen. FreCHEN (1993) widerspricht dieser Auffassung, da im Bereich der Schlackengrube des Tönchesberges weder verwitterte Tephren bzw. bodenüberprägte Sedimente noch warmzeitliche, pflanzenführende Horizonte gefunden werden. Statt dessen folgen auf die Tephraschichten eindeutig kaltzeitliche Ablagerungen aus vulkanoklastischem Gehängeschutt, unverwitterte Tephraablagerungen und Lösse. Nach BOENIGK \& FrECHEN (im Druck) gibt es in den Deckschichten keine Belege für interglaziale oder interstadiale Klimabedingungen direkt oberhalb der TönchesbergSchlacken. Diese Auffassung wird durch die Befunde von KOLFSCHOTEN \& ROTH (in Vorbereitung) unterstützt, die unterhalb des eemzeitlichen Bodens nur kaltzeitliche Molluskenarten finden, während oberhalb des Eembodens auch im Löß umgelagerte, warmzeitliche Mollusken in geringer Anzahl vertreten sind.

Da es sich bei diesen Vulkanausbrüchen um geologisch kurzzeitige Ereignisse handelt, ist für die Vulkane Tönchesberg, Plaidter Hummerich und Korretsberg ein kaltzeitliches (saalezeitliches) Eruptionsalter wahrscheinlich.

\subsection{Saale}

Der Begriff Saale wird in dieser Arbeit als Synonym für die vorletzte Kaltzeit verwendet. Aus Profil C wurden 10 TL-Proben bearbeitet (Abb. 3). 
Sedimentologie Pedologie

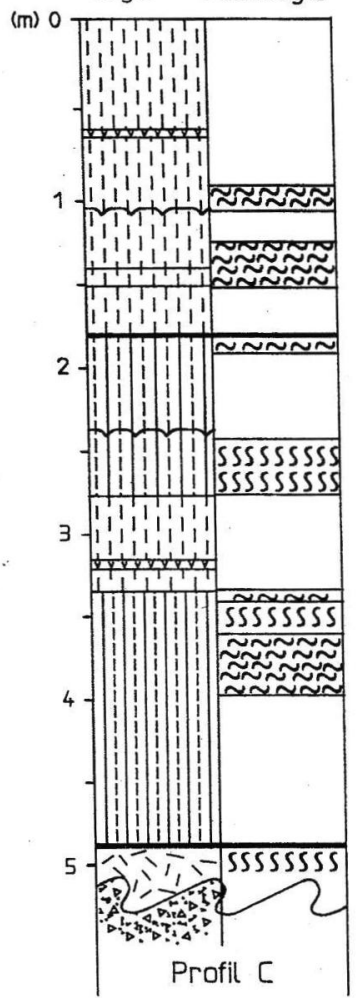

Ergebnisse der TL-Altersbestimmungen (Additive Methode)

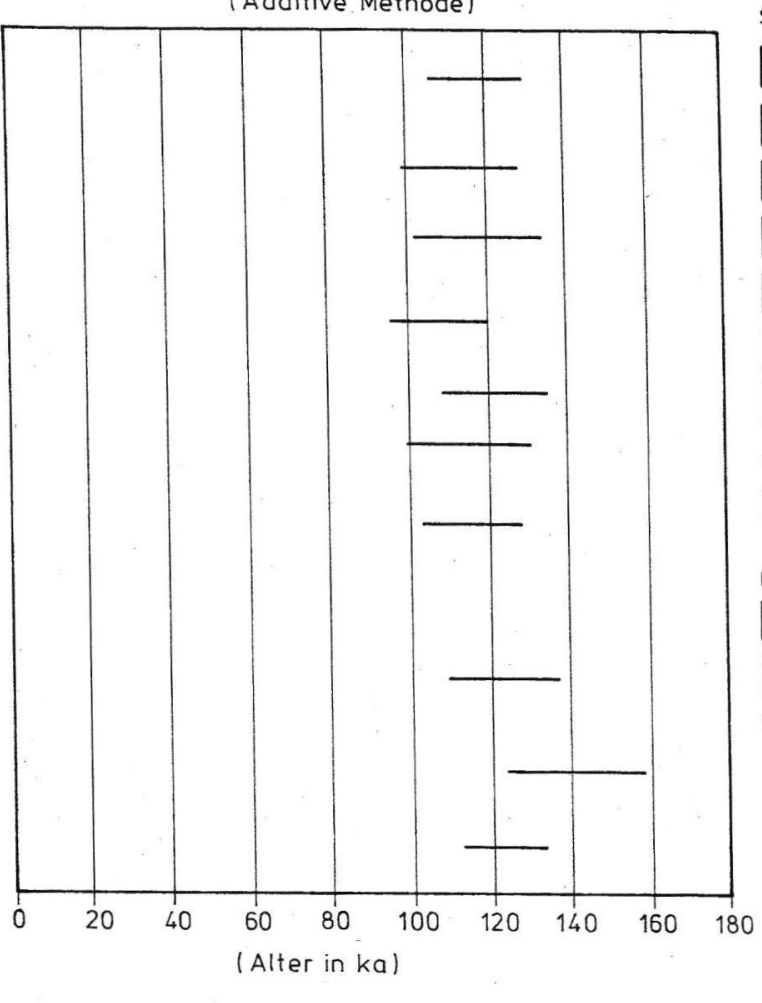

LEGENDE

Sedimentologie

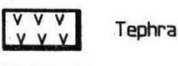

LOB

IIIIII LuBlehm

IIIII SchwemnltuB

L- F Fließerde

- Fließerde,

--Z Bodensediment verspolt

Diskordanz

Frostspalten

Pedologie :

$x \times x \times y$ Pararendzina

Parabraunerde

IIIIII Verbraunung

TIIIII Feuchtschwarzerde in situ, (Humuszone) Vernässungserscheinungen

Abb. 3: Thermolumineszenz-Datierungsergebnisse für die saalezeitliche Abfolge am Tönchesberg (Profil C). Die akkumulierte Dosis wurde mit der Additiven Methode bestimmt. Die TL-Alter zeigen keine Alterszunahme zum Liegenden hin.

Fig. 3: Results of thermoluminescence dating for loess of profile $\mathrm{C}$ from the penultimate glaciation showing no increase in age with depths for the Tönchesberg section. The additive dose method was used for determination of the equivalent dose.

Die TL-Daten lieferten für den vorletztglazialen Löß weder mit der Regenerierungs- noch mit der Additiven Methode eine Alterszunahme zum Liegenden hin. Aufgrund der typischen Wechsellagerung von Lössen mit Vernässungen und Naßböden wird die Abfolge in die jüngere Saale-Kaltzeit, Ober-Riß im Sinne von BIBus (1974: 168ff), gestellt. Unter der Voraussetzung, daß die marine Sauerstoffisotopen-Chronologie mit terrestrischen Ablagerungen vergleichbar ist, sind die TL-Analysenergebnisse mindestens um 25-30\% altersunterbestimmt. Das geologisch geschätzte Alter der saalezeitlichen Lösse reicht von 130 bis $150 \mathrm{ka}$.

Die TL-Daten, die mittels Additiver Methode ermittelt worden sind, stimmen numerisch am besten mit den geologisch geschätzten Altern überein (Abb. 3 und 6). Für den vorletztglazialen Löß wurden TL-Alter zwischen $141 \pm 18$ und $108 \pm 12$ ka kalkuliert. Allerdings nehmen die TL-Alter zum Liegenden hin nicht zu. Die Aussagefähigkeit der Daten der Additiven Methode ist somit nicht besser als die der Regenerierungs-Methode. Aus dem TL-Alter von $141 \pm 18$ ka kann nicht geschlossen werden, daß vorletztglaziale Lösse mit der TL datierbar sind. Die TL-Alter, die mit der Regenerierungs-Methode bestimmt wurden, liegen zwischen $118 \pm 9$ und $104 \pm 9 \mathrm{ka}$. Es ist keine Alterszunahme zum Liegenden hin erkennbar.

Sowohl mit der Regenerierungs-Methode als auch mit der Additiven Methode wurden beispielsweise in Ariendorf unterhalb der letztinterglazialen Parabraunerde TL-Alter von 140 bis $170 \mathrm{ka}$ bestimmt (FRECHEN 1990a: 115). Als Grund für die unterschiedlich hohen Datierungsobergrenzen wurden Langzeitfading oder verschieden große Sättigungseffekte angenommen.

Am Tönchesberg ist für die saalezeitlichen Lösse eine geologische Deutung der zum Liegenden hin fehlenden Alterszunahme möglich. Die Standardabweichung der TL-Alter beträgt etwa $10 \%$, also $\pm 10-20 \mathrm{ka}$ für die saalezeitlichen Lösse. Falls der saalezeitliche Löß beispielsweise nur innerhalb von 10 bis $20 \mathrm{ka} \mathrm{ak}$ kumuliert worden ist, läßt sich diese vorletztglaziale Lößakkumulationsphase altersmäßig mit den TL-Daten nicht auflösen (Abb. 6 und 7). Die Untersuchungen der Profile Ariendorf und Tönchesberg zeigen, 
daß die TL-Datierungsobergrenze von etwa 90 bis 120 ka für Lösse noch immer gilt, unabhängig davon, welche Datierungstechnik angewendet wird. Eine Einschätzung von TL-Altern als absolute Werte für Proben unterhalb des letztinterglazialen Bodens ist zur Zeit wegen fehlender systematischer und vergleichbarer Untersuchungen noch nicht erlaubt.

\subsection{Eem}

Der eemzeitliche Boden ist am Tönchesberg durch den Bt-Rest einer gekappten Parabraunerde vertreten. Mit der Thermolumineszenz-Methode kann nur das Sedimentationsalter von Lössen bestimmt werden. Der Zeitpunkt der Bodenbildung ist dagegen nicht datierbar. Für den Löß, aus dem sich die Parabraunerde entwickelte, ist somit ein saalezeitliches Sedimentationsalter zu erwarten.

Aus dem letztinterglazialen Boden wurden zwei Proben bearbeitet (Abb. 4).

\subsection{Unterweichsel}

Aus den umgelagerten Bodensedimenten wurden zwei Proben in Profil B genommen (Abb. 4). Die Sedimentationsalter liegen bei der RegenerierungsMethode zwischen $91 \pm 10$ und $86 \pm 0$ ka und bei der Additiven Methode zwischen $93 \pm 12$ und $89 \pm 12 \mathrm{ka}$.

Aus den Bodensedimenten wurde eine reverse Magnetisierung beschrieben, die als Blake Event gedeutet wird (Becker, Boenigk \& Hentzsch 1989: 20; REINDERS \& HAMBACH im Druck). Der Blake Event wird zeitlich an den Übergang der marinen SauerstoffIsotopenstadien von 5 e nach $5 \mathrm{~d}$ gestellt (TuchOlKA 1987: 320).

Da die Bodensedimente oberhalb der Parabraunerde dem klimatischen Übergang vom Klimaoptimum zu interstadialen Verhältnissen entsprechen, liegt eine Korrelation mit dem Blake Event nahe (BOENIGK \& FrFCHEN im Druck). Ein U/Th-Alter von $72 \pm 4$ ka (CHEN

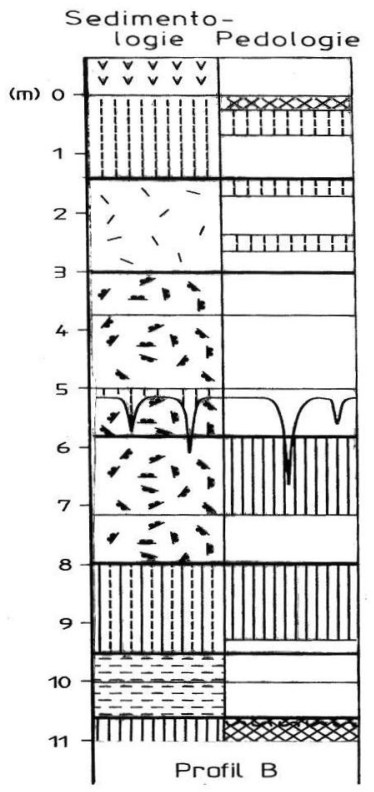

\section{Ergebnisse der TL-Altersbestimmungen \\ (Additive Methode)}

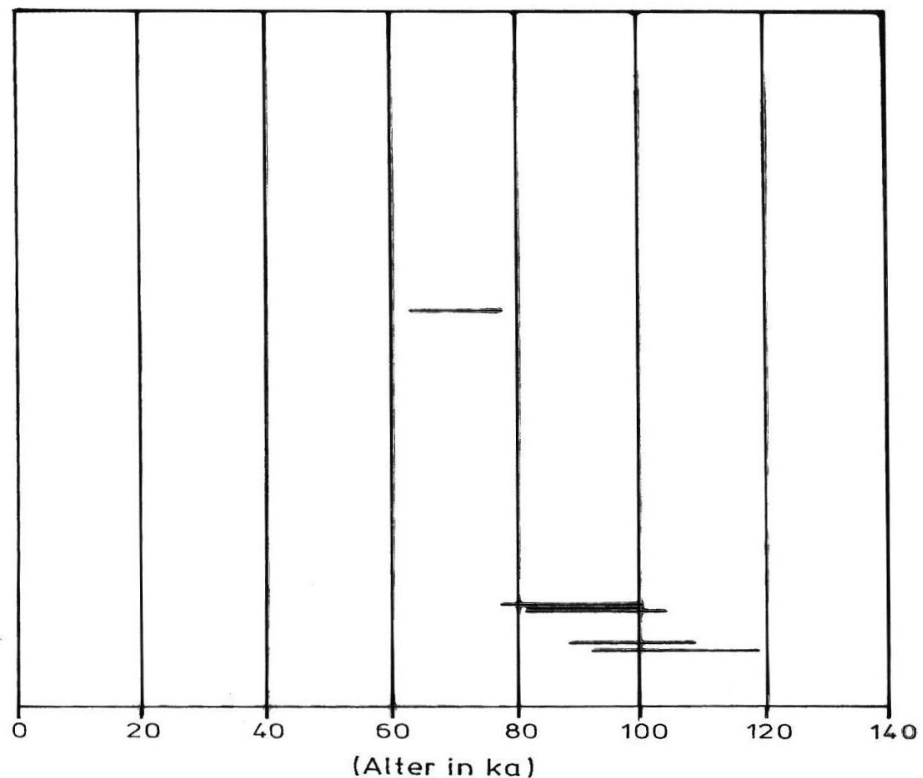

Abb. 4: Thermolumineszenz-Datierungsergebnisse für die eem- und weichselzeitliche Abfolge am Tönchesberg (Profil B). Die akkumulierte Dosis wurde mit der Additiven Methode bestimmt.

Fig. 4: Results of thermoluminescence dating for loess of profile B from the last glaciation. The additive dose method was used for determination of equivalent dose.

Die Sedimentationsalter des Lösses, aus dem die Parabraunerde entstanden ist, liegen zwischen $101 \pm 9$ und $97 \pm 13 \mathrm{ka}$ (REGEN) sowie $99 \pm 10$ und $106 \pm 13 \mathrm{ka}$ (ADD) (Abb. 4 und 6). Unter der Voraussetzung, daß das letztinterglaziale Klimaoptimum nach $127 \mathrm{ka}$ begann, sind für die TL-Alter beider Methoden Altersunterbestimmungen von mindestens $25-30 \%$ anzunehmen.
Tiemei, Beijing, aus Conard 1988: 23) ist mit den TLAltern und der lößstratigraphischen Interpretation allerdings kaum konsistent (BOENIGK \& FRECHEN im Druck).

Die Sedimente des Unterweichsels sind von einem mehrfachen Wechsel zwischen mäßig kalten Interstadialen und wesentlich kälteren Stadialen geprägt. 
Im Anschluß an die Umlagerung der Bodensedimente ist ein klimatischer Übergang von kalt-feuchten zu kalt-trockenen Bedingungen aus dem abnehmenden Grad der Verschwemmung des Lösses ableitbar (BOENIGK \& Frechen im Druck). Aus dem Schwemmlöß wurden 17 Proben aus Profil A analysiert (Abb. 5). untere Markerlöß wurden mit insgesamt fünf Proben bearbeitet (Abb. 4 und 5). Die TL-Alter für die in situHumuszonen und die humosen Fließerden liegen zwischen $84 \pm 9$ und $66 \pm 9 \mathrm{ka}$ (REGEN) und $71 \pm 8 \mathrm{ka}$ (ADD). Die Daten der pedogen überprägten Lösse und des Markerlösses sind stratigraphisch konsistent und stim-

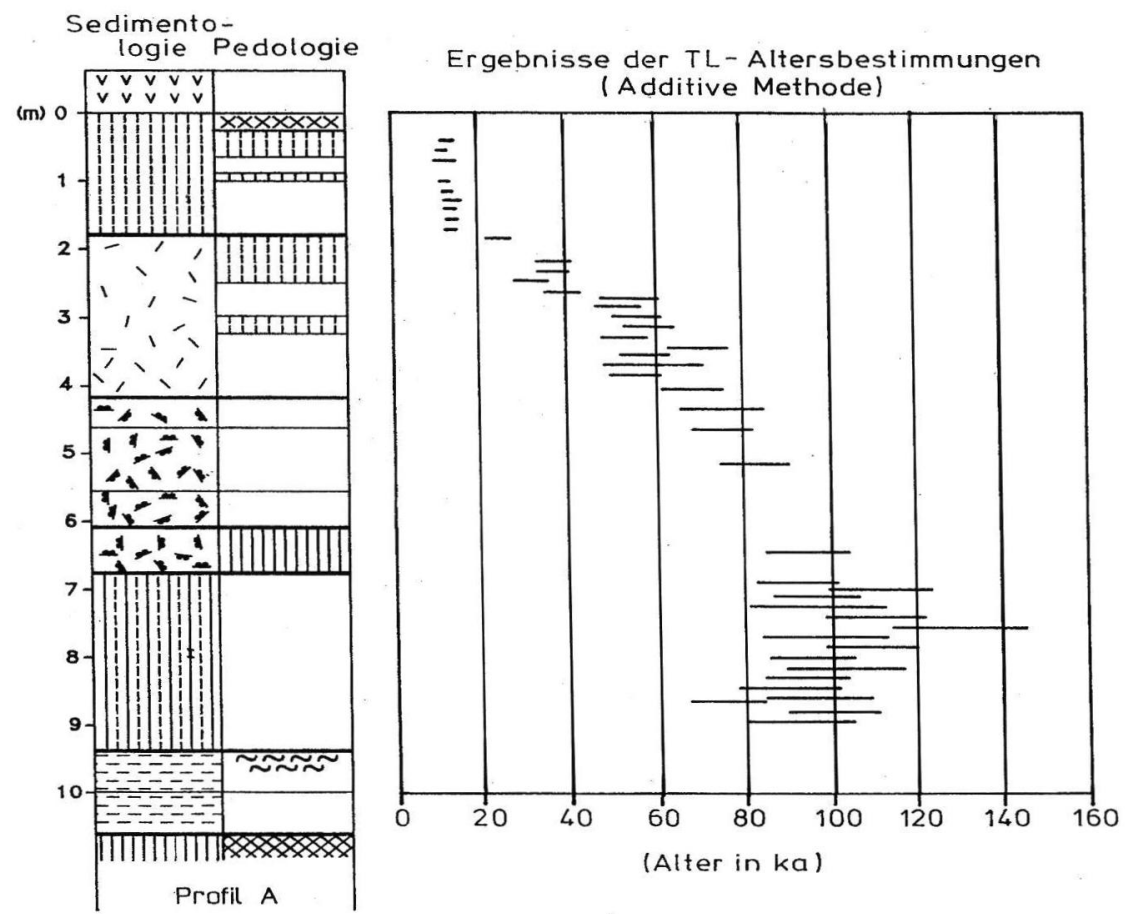

Abb. 5: Thermolumineszenz-Datierungsergebnisse für die würmzeitliche Abfolge am Tönchesberg (Profil A). Die akkumulierte Dosis wurde mit der Additiven Methode bestimmt.

Fig. 5: Results of thermoluminescence dating for loess of profile A from the last glaciation. The addive dose method was used for determination of equivalent dose.

Zusätzlich wurde eine Probe für einen Interlaborvergleich von 6 TL-Labors parallel analysiert (FrECHEN 1991a: 90). Die Sedimentationsalter des Schwemmlösses liegen zwischen $101 \pm 12$ und $70 \pm$ $7 \mathrm{ka}$ (REGEN) sowie zwischen $130 \pm 17$ und $76 \pm 9 \mathrm{ka}$ (ADD). Das geologisch geschätzte Alter liegt zwischen 110 und 100 ka v. H. Die überwiegende Anzahl der TL-Daten liegt darunter und läßt eine Altersunterbestimmung von mindestens 10\% erwarten.

Die Sedimentationsalter für den Schwemmlöß nehmen zum Liegenden hin nicht zu. Somit legt die Interpretation nahe, daß der Löß rasch akkumuliert worden ist. Die Sedimentationsphase war kürzer als das Mutungsintervall der TL-Daten und kann somit zeitlich nicht aufgelöst werden. Abgesehen von Ausnahmen stimmen die Sedimentationsalter von Regenerierungs- und Additiver Methode überein.

Im Anschluß an die erste Lößakkumulation kam es zu einem Wechsel von mehreren Bodenbildungsphasen unter interstadialen Verhältnissen und Umlagerungen. Die Waldsteppenböden, humosen Fließerden und der men mit den geologisch geschätzten Altern für diese Ablagerungen überein.

\subsection{Mittelweichsel}

Die Fließerden des Mittelweichsels wurden an 15 Proben aus Profil A untersucht (Abb. 5). Die Sedimentationsalter liegen zwischen $64 \pm 7$ und $36 \pm 4$ ka (REGEN) sowie $69 \pm 7$ und $32 \pm 4 \mathrm{ka}$ (ADD). Für die schwachen Verbraunungen innerhalb des Mittelweichsels lassen sich die Bodenbildungsphasen zeitlich eingrenzen. Die unterste Bodenbildung muß jünger als $54 \pm 6 \mathrm{ka}$ (REGEN) bzw. $53 \pm 6$ ka (ADD) sein. Die Dauer der Bodenbildung ist kleiner als die Standardabweichung, so daß sich eine Sedimentationsunterbrechung trotz hoher Probenzahl nicht auflösen läßt.

Die oberste Bodenbildung, die mit dem DenekampInterstadial korreliert wird, ist aufgrund der Sedimentationsalter des Lösses jünger als $36 \pm 4$ ka (REGEN) bzw. 32 3 ka (ADD). 


\subsection{Oberweichsel}

Es wurden 11 Proben aus Profil A bearbeitet (Abb. 5). Die unterste Probe mit TL-Altern von $21 \pm 3 \mathrm{ka}$ (REGEN) und $25 \pm 4$ ka (ADD) war vermutlich ein Mischalter. Nach den TL-Alter des Profils wurde der Löß relativ rasch akkumuliert. Die TL-Alter stimmen innerhalb der Mutungsintervalle überein. Die Lößakkumulation erfolgte zwischen $18 \pm 2$ ka und $14 \pm 2 \mathrm{ka}$ v. H. (REGEN) bzw. von $15 \pm 2 \mathrm{ka}$ und $12 \pm 1 \mathrm{ka}$ v. H. (ADD).

Legt man die Mittelwerte der TL-Alter zugrunde, so ergibt sich für den Oberweichsel-Löß eine durchschnittliche Akkumulationsrate von mindestens 0,58 $\mathrm{mm} / \mathrm{Jahr}$ (REGEN). Im Oberweichsel vorhandene Kiesschnüre deuten für diesen Zeitabschnitt auf einen Wechsel von Akkumulation und Erosion zum Nettetal hin. Dadurch wird die Aussage zur Akkumulationsrate relativiert. Der zwischengeschaltete schwache Naßboden dokumentiert zudem eine Sedimentationsunterbrechung, die so kurz war, daß sie mit TLDaten nicht auflösbar ist. Aus den TL-Altern folgt, daß die pedogene Überprägung im Alleröd-Interstadial jünger als $14 \pm 2 \mathrm{ka}$ (REGEN) bzw. 12 1 ka (ADD) ist, aber älter als 11 ka nach warvenchronologischen, ${ }^{14} \mathrm{C}$ und TL-Altern des Laacher See-Bimses (vgl. Bofnigk \& FRECHEN im Druck). Allerdings ist zu bedenken, daß die ${ }^{14} \mathrm{C}$-Zeitskala gegenüber der absoluten um etwa 1400 Jahre zu kurz ist (Becker, Kromer \& Triborn 1991).

\section{Zusammenfassung}

Die Gesamtinterpretation der Ergebnisse des Profils Tönchesberg aus der Osteifel zeigen, daß Lösse mit der Thermolumineszenz-Methode, auch bei systematischer Arbeitsweise und hoher Probenzahl, nur bis zu "absoluten" Altern von etwa 90 ka datierbar sind.

Datierungsobergrenzen von mehr als 100 ka lassen sich nur durch gezielte Anpassung der Meßbedingungen und -parameter erreichen, um den vermuteten geologischen Altersabschätzungen oder unabhängigen Daten gerecht werden zu können. Da dabei wichtige physikalische Faktoren vernachlässigt werden, sind die TL-Daten als fragwürdig und hypothetisch zu bewerten und nicht als mathematisch bewiesene, unabhängige physikalische Alter.

Durch die gleichzeitige Anwendung von Additiver Methode und Regenerierungs-Methode wurde die Verläßlichkeit der Daten beurteilbar (Abb. 6 und 7). Stichprobenartige Untersuchungen liefern dagegen oft nur stratigraphisch scheinbar konsistente, physikalische Alter, denen aber keine oder nur geringe chronostratigraphische Bedeutung zukommt.

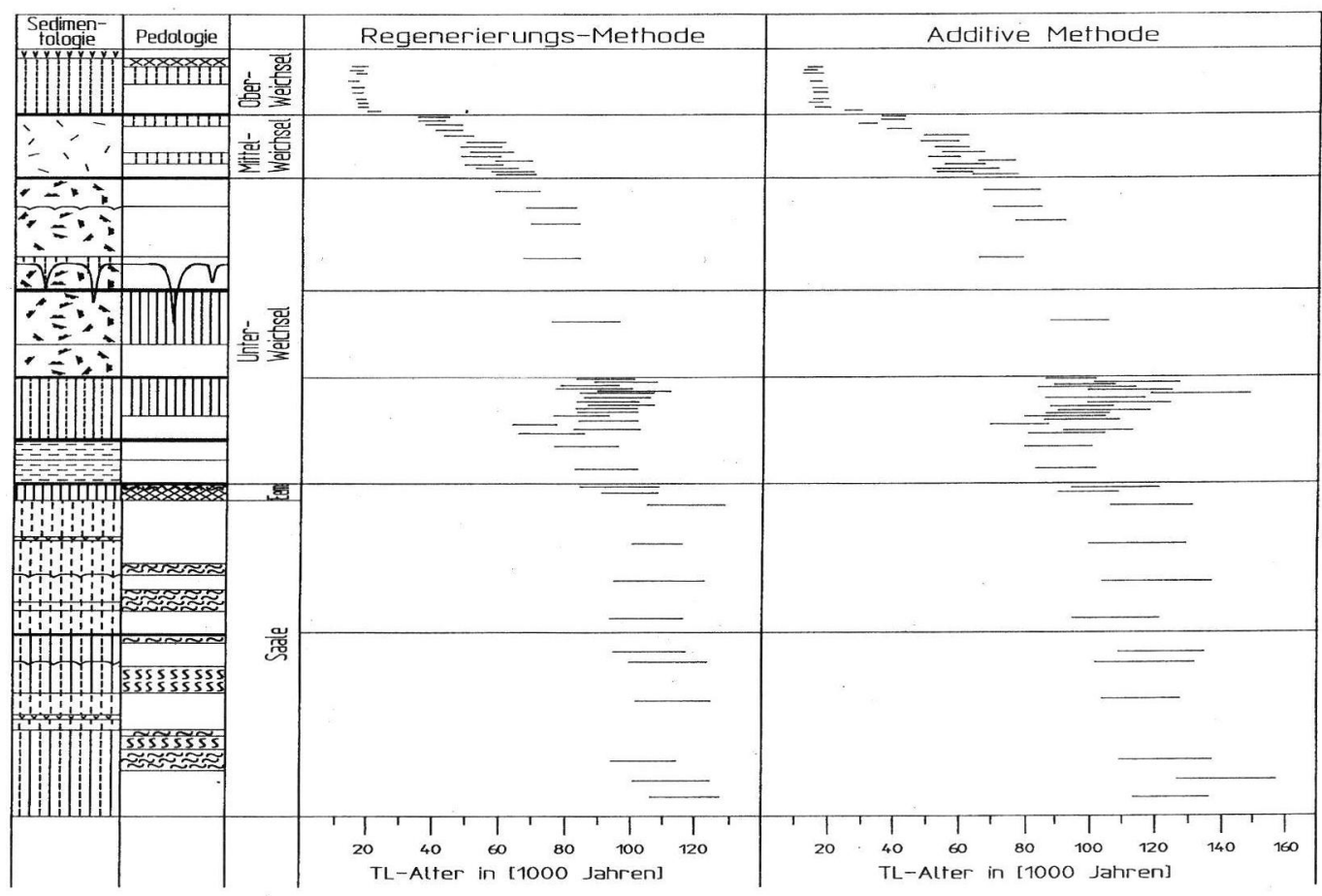

Abb. 6: Idealisiertes Profil der weichsel- und saalezeitlichen Löß-/Paläobodenabfolge des Tönchesberges mit einer Gegenüberstellung der mit der Regenerierungs-Methode und der Additiven Methode bestimmten TL-Alter.

Fig. 6: Idealised profile of the last and penultimated loess-/paleosol sequence of the Tönchesberg section. TL results were determined by regeneration method (REGEN) and additive dose method (ADD). 


\begin{tabular}{|c|c|c|c|c|c|c|}
\hline \multirow{2}{*}{$\begin{array}{l}\text { Sedimen- } \\
\text { tologie }\end{array}$} & \multirow{2}{*}{ Pedologie } & \multirow{2}{*}{ Gliederung } & \multirow{2}{*}{ Geologie } & \multicolumn{3}{|c|}{ Physikalische Alter in [1000 Jahren] } \\
\hline & & & & TL (REGEN) & TL (ADD) & $\mathrm{U} / \mathrm{Th} ;[-14 ; \mathrm{Ar} / \mathrm{Ar}$ \\
\hline & 피 & Ober-Weichsel & $\begin{array}{l}\text { Laacher See Birns } \\
\text { Allerud-Baden } \\
\text { LGB }\end{array}$ & $\begin{array}{r}14,1=1,5 \\
17,5 * 1,8\end{array}$ & $\begin{array}{l}12,0=1,2 \\
15,1=1,8\end{array}$ & {$[-14: 11,0$} \\
\hline $\begin{array}{l}1,-1 \\
1,1, \\
-1\end{array}$ & $\begin{array}{l}\text { साथाता } \\
\text { सापाप्य }\end{array}$ & Mittel-Weichsel & $\begin{array}{l}\text { Lohner Boden } \\
\text { Fließerde } \\
\text { unterer Lohner Boden ? } \\
\text { FlieBerde }\end{array}$ & $\begin{array}{l}36,5 \pm 3,8 \\
64,3 \pm 7,0\end{array}$ & $\begin{array}{l}32,3 \pm 3.8 \\
68,9 \pm 7,3\end{array}$ & \\
\hline & & & $\begin{array}{l}\text { humose Fließerde } \\
\text { Marker-LBB }\end{array}$ & $65.5 \pm 8.7$ & $75,6=9,8$ & \\
\hline & & . & Lehmbruckelsande & & & . \\
\hline & & & $\begin{array}{l}\text { Marker-LOB } \\
\text { humose Fließerde }\end{array}$ & $77,8 \neq 8,9$ & $70,3 \neq 7,4$ & \\
\hline & & Unter-Weichsel & Humuszone "in situ" & & & \\
\hline & & & humose Fließerde & $84,3 \pm 8,9$ & $95,2 \pm 9,7$ & \\
\hline & & & $\begin{array}{l}\text { Humuszone "in situ" } \\
\text { SchwemmloB }\end{array}$ & $\begin{array}{c}84,1 \pm 9,2 \\
101 \pm 11\end{array}$ & $\begin{aligned} 90,5 & \pm 11,2 \\
112 & \pm 12\end{aligned}$ & \\
\hline & & & ungelagertes & $85,7 \pm 9,8$ & $89,0 \pm 11,5$ & \\
\hline$E=-==--1$ & & & Bodensediment & $90,7 \pm 9,6$ & $92,9 \pm 11,5$ & $\begin{array}{l}\text { Blake Event: } 110 \\
\text { u/Th: } 72 \pm 4\end{array}$ \\
\hline मागय & scos & Eern & Parabraunerde & $100 \pm 9,2$ & $106 \pm 13$ & \\
\hline 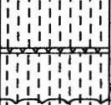 & & & $\begin{array}{l}\text { LÖB } \\
\text { Tephra }\end{array}$ & $116 \pm 13$ & $118 \pm 12$ & \\
\hline 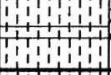 & 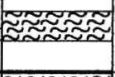 & Saale & LUB mit Vernăssungen & $104 \pm 12$ & $108 \pm 12$ & \\
\hline & 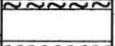 & & $\begin{array}{l}\text { SchwemmluB mil Ver- } \\
\text { nässungen }\end{array}$ & $106 \pm 11$ & $122 \pm 13$ & \\
\hline & 555555555 & & NaBboden & & & \\
\hline 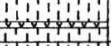 & & & Tephra & & & \\
\hline & 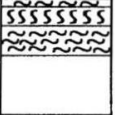 & & $\begin{array}{l}\text { NaBboden } \\
\text { SchwernmluB mit Ver- } \\
\text { nässungen }\end{array}$ & $118 \neq 9$ & $\begin{array}{c}(141 \pm 17) \\
123 \pm 10\end{array}$ & \\
\hline 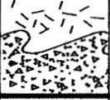 & sss5s5ss & & $\begin{array}{l}\text { Fließerde mit NaBboden } \\
\text { vulkanoklastischer Detritus }\end{array}$ & & & \\
\hline 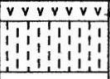 & & & $\begin{array}{l}\text { tephritische Tephra } \\
\text { LuB mit Vernässung }\end{array}$ & & & \\
\hline 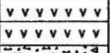 & & & basanilische Tephren & & & $\begin{array}{l}\text { Ar/Ar: } 238=20 \\
\text { Ar/Ar: } 243 \neq 65\end{array}$ \\
\hline Pus & & & $\begin{array}{l}\text { vulkanische Schlacken, } \\
\text { anstehend }\end{array}$ & & & $A \Gamma / A \Gamma: 202 \pm 14$ \\
\hline
\end{tabular}

Abb. 7: Idealisiertes Profil der Löß-/Paläobodenabfolge des Tönchesberges. Vergleich der Datierungsergebnisse der unterschiedlichen Methoden. ${ }^{40} \mathrm{Ar} /{ }^{39} \mathrm{Ar}$-Datierungen nach BOGAARD \& SCHMINCKE $(1990),{ }^{40} \mathrm{~K} /{ }^{40} \mathrm{Ar}-\mathrm{Datierungen}$ nach SCHMINCKE \& MerTes (1979), ${ }^{14}$ C-Alter zusammengestellt nach BOGAARD \& SCHMINCKE (1985), U/Th-Datierungen nach CONARD (1988) und die inverse Magnetisierung nach BECKER, BOENIGK \& HENTZsCH (1989).

Fig. 7: Idealised profile of the loess palaeosol sequence of section Tönchesberg. Comparison of dating results by different methods. ${ }^{40} \mathrm{Ar} /{ }^{39} \mathrm{Ar}$-dating by BOGAARD \& SCHMINCKE (1990), ${ }^{70} \mathrm{~K} /{ }^{10} \mathrm{Ar}$-dating by SCHMINCKE \& MERTES (1979), ${ }^{14} \mathrm{C}$ ages compiled by BOGAARD \& SCHMINCKE (1985), uranium thorium dating mentioned by CONARD (1988) and reverse magnetization by BECKER, BOENIGK \& HENTZSCH (1989).

Für den Tönchesberg liegen ${ }^{40} \mathrm{Ar} /{ }^{39} \mathrm{Ar}$ - und ${ }^{40} \mathrm{~K} /{ }^{40} \mathrm{Ar}$ Daten der Tönchesberg-Schlacken und Tephren des Plaidter Hummerichs sowie Korretsberges vor. Sie sind mit etwa $200 \mathrm{ka}$ als Maximalalter für die Ausbrüche der drei Schlackenkegel zu deuten. Aus lößstratigraphischer Sicht sind sie zu hoch, da keinerlei Hinweise für interglaziale oder interstadiale Klimabedingungen direkt oberhalb der TönchesbergSchlacken vorliegen. Auf die Tephraschichten folgen eindeutig kaltzeitliche Ablagerungen, so daß ein kaltzeitliches Eruptionsalter wahrscheinlich ist.

Für die Lösse unterhalb der Parabraunerde wird aufgrund der typischen Wechsellagerung von Lössen mit Naßböden und Vernässungen eine Sedimentation während der jüngeren Saale angenommen. Die TLDaten unterstützen diese Auffassung nicht, weil für die vorletztglazialen Lösse zum Liegenden hin keine Alterszunahme erkennbar ist. Neben methodischen 
Gründen, Langzeitfading und TL-Sättigung, kann als Grund eine im Vergleich zur Standardabweichung kurze Dauer der Lößsedimentationsphase von weniger als $20 \mathrm{ka}$ angenommen werden.

Eine zeitlich genauere Eingrenzung des letztinterglazialen Bodens kann wegen der TL-Altersunterbestimmung nicht erfolgen. Auch in den Schwemmlössen oberhalb des Bt-Rests der Parabraunerde nehmen die TL-Alter zum Liegenden hin nicht sichtbar zu. Als Grund wird eine mit der TL-Methode nicht auflösbare zu kurze Zeitspanne der Lößakkumulation angenommen. Im Anschluß an diese letztglaziale LößSedimentationsphase kam es zu einem Wechsel von Bodenbildungsphasen unter interstadialen Verhältnissen und Umlagerungen.

Innerhalb des Mittelweichsels herrschen Fließerden vor, die durch zwei schwache interstadiale Bodenbildungen untergliedert sind. Die Sedimentationsalter der Lösse liegen zwischen $32 \pm 4$ und $70 \pm 8 \mathrm{ka}$. Die Bodenbildungsphasen sind kürzer als die Standardabweichungen der TL-Alter, so daß die Sedimentationsunterbrechungen trotz hoher Probenzahl nicht aufgelöst werden können.

Für die oberwürmzeitlichen Lösse ist auch eine rasche Akkumulation anzunehmen, da die TL-Alter innerhalb der Mutungsintervalle übereinstimmen. Aufgrund der TL-Alter liegt der Schluß nahe, daß die pedogene Überprägung während des Alleröds nach $14 \pm 2$ ka (REGEN) bzw. $12 \pm 1$ ka (ADD), aber vor dem Ausbruch des Laacher Sees vor 12,5 ka erfolgte.

\section{Dank}

Für die ausgiebige und fruchtbare Diskussion möchte ich mich bei Herrn Prof. Dr. W. Boenigk und den Mitarbeitern der Abteilung Quartärgeologie der Universität zu Köln bedanken. Herrn Prof. Dr. M. A. Geyh sei Dank für die kritische Durchsicht des Manuskriptes. Des weiteren danke ich der Deutschen Forschungsgemeinschaft (Bo 413/5-3) für die gewährte finanzielle Unterstützung, und der Fritz Thyssen Stiftung für die Bereitstellung von Mitteln zum Aufbau eines Lumineszenz-Labors im Geologischen Institut der Universität zu Köln.

\section{Schriftenverzeichnis}

AitKen, M. J. (1985): Thermoluminescence Dating. - 1-359, Academic Press; London.

AKTAS, A. \& FreCheN, M. (1991): Mittel- und jungpleistozäne Sedimente der Hochterrassen in der nördlichen IllerLech-Platte. - Geologisches Institut der Universität zu Köln, Sonderveröffentlichungen, 82 (Festschrift Karl Brunnacker): 19-41; Köln.

BAlescu, S. (1988): Apports de la thermoluminescence à la stratigraphie et à la sédimentologie des loess du NordOuest de l'Europe. - Dissertation, Universität Brüssel, Vol I: 1-199 (unveröffentlicht); Brüssel.
Becker, U., Boenigk, W. \& Hentzsch, B. (1989): Reverse Magnetisierung in den frühwürmzeitlichen Deckschichten am Tönchesberg/Osteifel. - Mainzer Naturw. Archiv, 27: 1-22; Mainz.

Becker, B., Kromer, B. \& Trimborn, P. (1991): A stable-isotope tree-ring timescale of the Late Glacial/Holocene boundary. - Nature, 353: 647-649; London.

BerGER, G. W. (1990): Effectiveness of natural zeroing of the thermoluminescence in sediments. - Journal of Geophysical Research, 95, B8: 12375-12397; Washington.

Berger, G. W., Pillans, B. J. \& Palmer, A. S. (1992): Dating loess up to 800 ka by thermoluminescence. - Geology, 20: 403-406; Boulder, Colorado.

Bıbus, E. (1974): Abtragungs- und Bodenbildungsphasen im Rißßöß. - Eiszeitalter und Gegenwart, 25: 166-182; Öhringen.

BOENIGK, W. \& FreChen, M. (1991): Chronostratigraphy of eolian sediments from Germany and its paleoclimatic application. - XIII INQUA Congress in Beijing, China, Abstracts, p. 29; Beijing.

- (im Druck): Mittel- und oberpleistozäne Deckschichten des Tönchesberges, Osteifel. - In: Schirmer, W. (Hrsg.): Landschaftsgeschichte im europäischen Rheinland. - Rheinland-Verlag, Köln.

BogaARD, P. v. d. \& SCHMincke, H.-U. (1985): Laacher See Tephra: A widespread isochronous late Quaternary tephra layer in central and northern Europe. - Geological Society of America Bulletin, 96: 1554-1571; Boulder.

- (1990): Die Entwicklungsgeschichte des Mittelrheinraumes und die Eruptionsgeschichte des Osteifel-Vulkanfeldes. - In: SchIrMFr, W. (Hrsg.): Rheingeschichte zwischen Mosel und Maas. - DEUQUA Führer 1: 166190; Hannover.

BuschbeCk, H. M., Chun, H.-U,, DÖNIG, R. \& Gessler, R. (1992): Thermoluminescence dating of loess horizons in Wiesbaden-Gräselberg and Wallertheim by the quartzinclusion method. - Quaternary Science Reviews, 11: 19-23; Oxford.

CONARD, N. J. (1988): Excavations at Tönchesberg, a Middle Paleolithic site in the central Rhine valley, - Yale Graduate Journal of Anthropology, 1: 21-36; New Haven.

DeBenham, N. C. (1985): Use of UV emissions in TL dating of sediments. - Nuclear Tracks and Radiation Measurements, 10: 717-724; Oxford.

FRECHEN, M. (1990a): TL-Datierungen in Ariendorf. - In: SCHIRMER, W. (Hrsg.): Rheingeschichte zwischen Mosel und Maas. - DEUQUA Führer 1: 114-117; Hannover.

- (1990b): TL-Datierungen am Tönchesberg. In: ScHIRMER, W. (Hrsg.): Rheingeschichte zwischen Mosel und Maas. - DEUQUA Führer 1: 56-57; Hannover.

- (1991a): Thermolumineszenz-Datierungen an Lössen des Mittelrheingebiets. - Geologisches Institut der Universität zu Köln, Sonderveröffentlichungen, 79: 1-137; Köln

- (1991b): Interpretation physikalischer Datierungen in der Quartärforschung. - Geologisches Institut der Universität zu Köln, Sonderveröffentlichungen, 82 (Festschrift Karl Brunnacker): 99-112; Köln.

- (1992): Systematic thermoluminescence dating of two loess profiles from the Middle Rhine Area (F. R. G.). Quaternary Science Reviews, 11: 93-101; Oxford.

- (1993): Eruptionsgeschichte und Deckschichtenfolge des Schlackenkegelkomplexes der Wannenköpfe in der 
Osteifel. - Nachrichten der Deutschen Geologischen Gesellschaft, 50: 114-115; Hannover.

FreChen, M., BrÜCKNER, H. \& RADTKE, U. (1992): A comparison of different TL-techniques on loess samples from Rheindahlen (F. R. G.). - Quarternary Science Reviews, 11: 109-113; Oxford,

GEYH, M. A. (1991): Determination of absolute dates for terrestrial materials (Last Interglacial to the Holocene). An appeal for careful interpretation. - In: FrENZEL, B. (Hrsg.): Klimageschichtliche Probleme der letzten 130000 Jahre. - Gustav Fischer Verlag; 251-265; Stuttgart.

HentzsCH, B. (1990): Die Lößdeckschichten am Tönchesberg (Osteifel). - In SCHIRMER, W. (Hrsg.): Rheingeschichte zwischen Mosel und Maas. - DEUQUA Führer 1: 42-46; Hannover.

JANNOTTA, A. (1991): Thermolumineszenzdatierungen als chronometrischer Beitrag zur stratigraphischen Beschreibung von Lößprofilen. - Düsseldorfer Geographische Schriften, 30: 1-116; Düsseldorf.

Juvigne, E. H. \& Wintle, A. G. (1988): A new chronostratigraphy of the late Weichselian loess units in Middle Europe based on thermoluminescence dating. - Eiszeitalter und Gegenwart, 38: 94-105; Hannover.

Kolfschoten, T., v. \& Roth, G. (in Vorbereitung): Mittelund spätpleistozäne Mollusken und Kleinsäuger aus Vulkanfüllungen des Osteifel-Gebietes, Deutschland. -

PACKMAN, S. C. \& GRÜN, R. (1992): TL analysis of loess samples from Achenheim. - Quaternary Science Reviews, 11: 103-107; Oxford.

REINIERS, J. \& HAMBACH, U. (im Druck): A geomagnetic event recorded in loess deposits of the Tönchesberg (East Eifel Volcanic Field, Germany). - Geophysical Journal International; London.

Rendell, H. M. \& Townsend, P. D. (1988): Thermoluminescence dating of a $10 \mathrm{~m}$ loess profile in Pakistan. - Quaternary Science Reviews, 7: 252-255; Oxford.

SCHMINCKE, H.-U. \& MERTES, H. (1979): Pliocene and Quaternary volcanic phases in the Eifel volcanic field. Naturwissenschaften, 66: 614-615; Heidelberg.
Schönhals, E., Rohdenburg, H. \& Semmel, A. (1964): Ergebnisse neuerer Untersuchungen zur Würmlöß-Gliederung in Hessen. - Eiszeitalter und Gegenwart, 15: 199206; Öhringen.

Tucholka, P., Fontugne, M., Gutchard, F. \& Paterne, M. (1987): The Blake magnetic polarity episode in cores from the Mediterranean Sea. - Earth Planetary Science Letters, 86: 320-326; Amsterdam.

WinTLE, A. G. (1985): Stability of the TL signal in fine grains from loess. - Nuclear Tracks and Radiation Measurements, 10: 725-730; Oxford.

-. (1990): A review of current research on TL dating of loess. - Quaternary Science Reviews, 9: 385-397; Oxford. - \& BRUNNACKER, K. (1982): Ages of volcanic tuff in Rheinhessen obtained by thermoluminescence dating of loess. - Die Naturwissenschaften, 69: 181-182; Heidelberg. - \& HunTLEY, D. J. (1982): Thermoluminescence dating of sediments. - Quaternary Science Reviews, 1: 31-53; Oxford.

- \& Packman, S. C. (1988): Thermoluminescence ages for three sections in Hungary. - Quarternary Science Reviews, 7: 315-320; Oxford.

- Questiaux, D. G., Roberts, R. G. \& Spooner, N. A. (1993): Comments on "Dating loess up to $800 \mathrm{ka}$ by thermoluminescence", - Geology, 21: 568-569; Boulder, Colorado.

XIE, J. \& AlTkEn, M. J. (1991): The hypothesis of mid-term fading and its trial on Chinese loess. - Ancient TL, 9: 2125; Durham.

ZÖller, L., CONARD, N. J. \& HAHN, J. (1991): Thermoluminescence dating of Middle Palaeolithic open air sites in the Middle Rhine Valley/Germany. - Naturwissenschaften, 78: 408-410; Heidelberg.

ZÖller, L, Stremme, H. \& Wagner, G. A. (1988): Thermolumineszenz-Datierung an Löß-Paläoböden-Sequenzen von Nieder-, Mittel- und Oberrhein. - Chem. Geology (Isot. Geosc. Sect.), 73: 39-62; Heidelberg.

Manuskript eingegangen am 26. 5. 1993 